\title{
Molecular gas and star formation toward the IR dust bubble S 24 and its environs $\star$
}

\author{
C. E. Cappa ${ }^{1,2}$, N. Duronea ${ }^{1}$, V. Firpo ${ }^{3}$, J. Vasquez ${ }^{1,2}$, C. H. López-Caraballo ${ }^{3}$, M. Rubio ${ }^{4}$, and M. M. Vazzano ${ }^{2}$ \\ 1 Instituto Argentino de Radioastronomía, CONICET, CCT La PLata, C.C.5, 1894 Villa Elisa, Argentina \\ e-mail: ccappa@fcaglp.unlp.edu.ar \\ 2 Facultad de Ciencias Astronómicas y Geofísicas, Universidad Nacional de la Plata, Paseo del Bosque s/n, 1900 La Plata, Argentina \\ 3 Departamento de Física y Astronomía, Universidad de La Serena, La Serena, Chile \\ ${ }^{4}$ Departamento de Astronomía, Universidad de Chile, 1058 Santiago, Chile
}

Received 23 February 2015 / Accepted 31 August 2015

\begin{abstract}
Aims. We present a multiwavelength analysis of the infrared dust bubble S 24 and the extended IR sources G341.220-0.213 and G341.217-0.237 located in its environs. We aim to investigate the characteristics of the molecular gas and the interstellar dust linked to them and analyze the evolutionary state of the young stellar objects identified there and the relation of the bubble to S 24 and the IR sources.

Methods. Using the APEX telescope, we mapped the molecular emission in the $\mathrm{CO}(2-1),{ }^{13} \mathrm{CO}(2-1), \mathrm{C}^{18} \mathrm{O}(2-1)$, and ${ }^{13} \mathrm{CO}(3-2)$ lines in a region of about $5^{\prime} \times 5^{\prime}$ in size around the bubble. The cold dust distribution was analyzed using submillimeter continuum images from ATLASGAL and Herschel. Complementary IR and radio data at different wavelengths were used to complete the study of the interstellar medium in the region.

Results. The molecular gas distribution shows that gas linked to the S 24 bubble and to G341.220-0.213 and G341.217-0.237 has velocities of between $-48.0 \mathrm{~km} \mathrm{~s}^{-1}$ and $-40.0 \mathrm{~km} \mathrm{~s}^{-1}$, compatible with the kinematical distance of $3.7 \mathrm{kpc}$ that is generally adopted for the region. The gas distribution reveals a shell-like molecular structure of $\sim 0.8 \mathrm{pc}$ in radius bordering the $\mathrm{S} 24$ bubble. A cold dust counterpart of the shell is detected in the LABOCA and Herschel-SPIRE images. The weak extended emission at $24 \mu \mathrm{m}$ from warm dust and radio continuum emission projected inside the bubble indicates exciting sources and that the bubble is a compact HII region. Part of the molecular gas bordering the S 24 HII region coincides with the extended infrared dust cloud SDC341.1940.221 . A molecular and cold dust clump is present at the interface between the S 24 HII region and G341.217-0.237, shaping the eastern border of the IR bubble. The arc-like molecular structure encircling the northern and eastern sections of the IR source G341.220-0.213 indicates that the source is interacting with the molecular gas. The analysis of the available IR point source catalogs reveals some young stellar object candidates linked to the IR-extended sources, thus confirming their nature as active star-forming regions. Gas and dust masses were estimated for the different features. The total gas mass in the region and the $\mathrm{H}_{2}$ ambient density amount to $10300 M_{\odot}$ and $5900 \mathrm{~cm}^{-3}$, indicating that G341.220-0.213, G341.217-0.237, and the S 24 HII region are evolving in a high-density medium. A triggering star formation scenario for the HII region is investigated.
\end{abstract}

Key words. molecular data - H II regions - submillimeter: ISM - ISM: molecules

\section{Introduction}

Churchwell et al. $(2006,2007)$ identified more than 600 infrared (IR) dust bubbles in the Spitzer $8.0 \mu$ m-images of the Galactic Legacy Infrared Mid-Plane Survey Extraordinaire (GLIMPSE, Benjamin et al. 2003) between longitudes of $-60^{\circ}$ to $+60^{\circ}$. These IR dust bubbles are bright at $8.0 \mu \mathrm{m}$, showing the emission of polycyclic aromatic hydrocarbons (PAHs) typical of photodissociation regions (PDRs), and enclose $24 \mu \mathrm{m}$ emission, indicating the existence of warm dust inside (Watson et al. 2008). These characteristics indicate excitation sources (Oand/or B-type stars) that dissociate the molecular gas in the border of the PDRs and heat the dust through their UV photons. The dense shells surrounding many IR dust bubbles are believed to favor the formation of new generations of stars through the

* Final reduced APEX data cube in FITS format is only available at the CDS via anonymous ftp to

cdsarc.u-strasbg.fr (130.79.128.5) or via

http://cdsarc.u-strasbg.fr/viz-bin/qcat?]/A+A/585/A30 radiatively driven implosion (RDI) or the collect-and-collapse (CC) processes (Pomarès et al. 2009; Deharveng et al. 2009, 2012; Samal et al. 2014). Indeed, detailed studies of these bubbles have shown young stellar objects (YSOs) in their environs, although in some cases triggered star formation could not be proved (see, for example, Alexander et al. 2013; Dewangan \& Ojha 2013).

We selected the complex of IR dust bubbles S 21-S 24 identified by Churchwell et al. (2006) in the GLIMPSE images to perform a multiwavelength study of the interaction with their environs and search for YSOs in their vicinity. Here we report on the study of S 24. This bubble is centered at RA, Dec $(\mathbf{J} 2000)=$ $\left(16^{\mathrm{h}} 52^{\mathrm{m}} 20^{\mathrm{s}},-44^{\circ} 28^{\prime} 10^{\prime \prime}\right)$ or $(l, b)=\left(341^{\circ} 203,-0^{\circ} 23\right)$ and coincides with the IR source IRAS 16487-4423, which has colors typical of ultracompact HII regions (Wood \& Churchwell 1989).

A composite image of S 24 and its environs at 3.6, 4.5, and $8.0 \mu \mathrm{m}$ is displayed in Fig. 1. At $3.6 \mu \mathrm{m}$ the emission arises in a faint PAH feature at $3.3 \mu \mathrm{m}$ and in dispersed stellar light, 


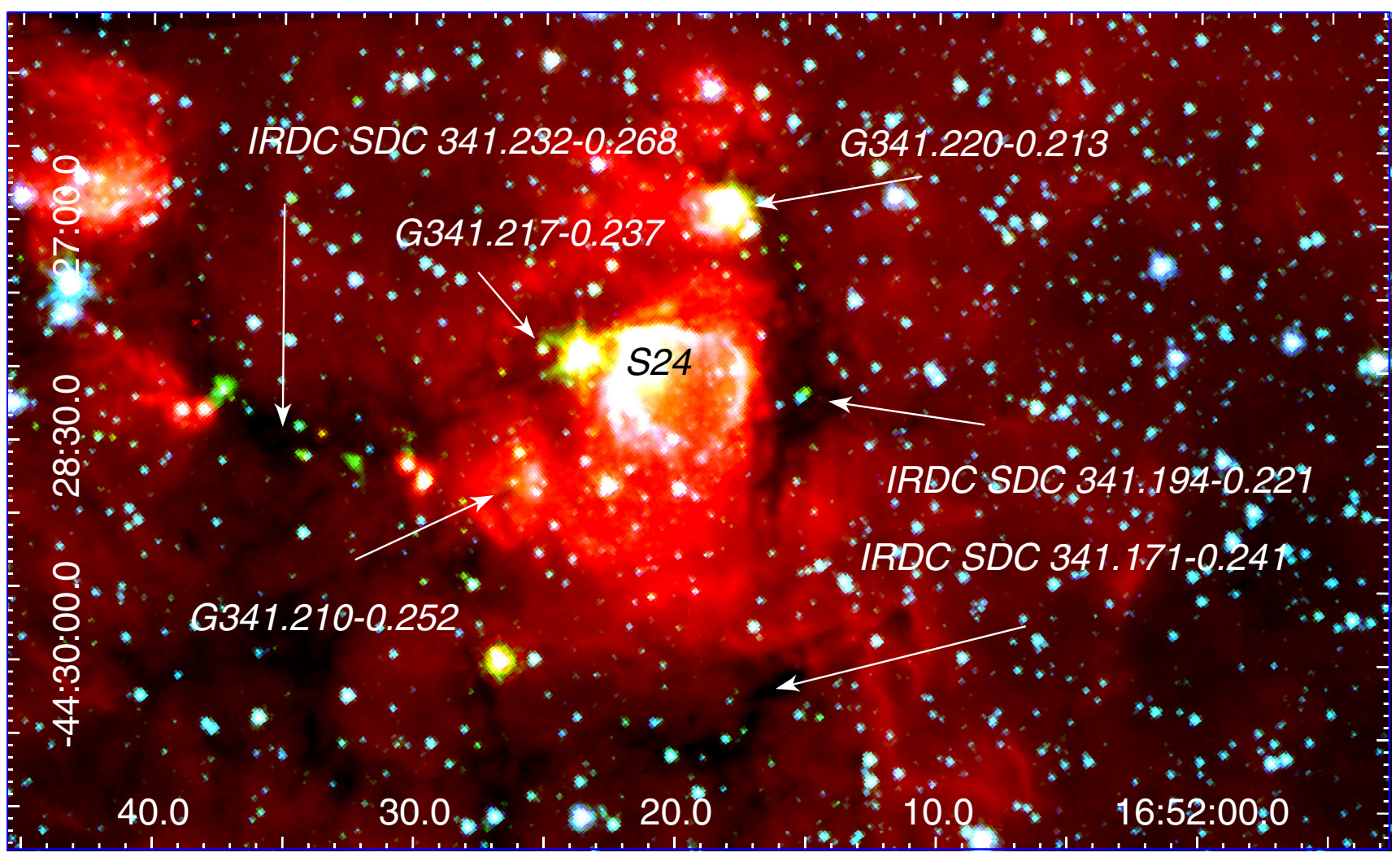

Fig. 1. Composite image of the IR dust bubble $\mathrm{S} 24$ and its environs showing IRAC images: $3.6 \mu \mathrm{m}$ is in blue, $4.5 \mu \mathrm{m}$ is in green, and $8.0 \mu \mathrm{m}$ is in red. The different features in the environment of S 24 are indicated.

while at $8.0 \mu \mathrm{m}$ most of the emission originates in strong features from PAHs. The $4.5 \mu \mathrm{m}$ band shows emission from $\mathrm{Br} \alpha$ and $\operatorname{Pf} \beta$, and vibrational $\mathrm{H}_{2}$ lines and ro-vibrational $\mathrm{CO}$ lines, typical of shocked gas (see Watson et al. 2008). At $8 \mu \mathrm{m}$ the bubble presents a full filamentary shell of about $28^{\prime \prime}$ in radius, brighter toward the north and east, and displays a sharp eastern border. Two small and bright regions of extended emission can be identified at $8 \mu \mathrm{m}$ in the image, named G341.2200.213 at RA, $\operatorname{Dec}(\mathrm{J} 2000)=\left(16^{\mathrm{h}} 52^{\mathrm{m}} 18.25^{\mathrm{s}},-44^{\circ} 26^{\prime} 54^{\prime \prime}\right)$, of about $10^{\prime \prime}$ in radius, and G341.217-0.237 at RA, Dec $(\mathrm{J} 2000)=$ $\left(16^{\mathrm{h}} 52^{\mathrm{m}} 23.74^{\mathrm{s}},-44^{\circ} 27^{\prime} 53^{\prime \prime}\right)$, of about $5^{\prime \prime}$ in radius, placed to the north and east of S 24, respectively. At this wavelength, the shape of G341.220-0.213 resembles a horseshoe opened toward the east. The infrared dark cloud (IRDC) identified by Peretto \& Fuller (2009) as SDC341.194-0.221 is evident to the west of the bubble where the emission at $8 \mu \mathrm{m}$ is lacking. It is centered at RA, Dec $(\mathrm{J} 2000)=\left(16^{\mathrm{h}} 52^{\mathrm{m}} 15.5^{\mathrm{s}},-44^{\circ} 28^{\prime} 19.4^{\prime \prime}\right)$ and extends almost $2^{\prime}$ in declination. Two IRDCs were also identified by Peretto \& Fuller (2009): one at RA, Dec $(J 2000)=\left(16^{\mathrm{h}} 52^{\mathrm{m}} 15.4^{\mathrm{s}}\right.$, $-44^{\circ} 30^{\prime} 6.9^{\prime \prime}$ ) (SDC 341.171-0.241), to the south of the bubble, and the other one at RA, Dec $(\mathrm{J} 2000)=\left(16^{\mathrm{h}} 52^{\mathrm{m}} 35.7^{\mathrm{s}}\right.$, $-44^{\circ} 28^{\prime} 21.8^{\prime \prime}$ ) (IRDC SDC 341.232-0.268). Finally, fainter emission can be detected in the whole region, encircling S 24, G341.22-0.213, and G341.217-0.237. An almost circular structure of about $16^{\prime \prime}$ in radius that we call G341.210-0.252, is also detected in the $8 \mu \mathrm{m}$ image at RA, Dec $(\mathrm{J} 2000)=\left(16^{\mathrm{h}} 52^{\mathrm{m}} 26.5^{\mathrm{s}}\right.$, $\left.-44^{\circ} 28^{\prime} 50^{\prime \prime}\right)$, to the southeast of S 24 . These features are indicated in Fig. 1. The figure also shows that G341.220-0.213, G341.217-0.237, and part of the S 24 bubble coincide with sources at $4.5 \mu \mathrm{m}$.

Henning et al. (2001) observed the region at $1.2 \mathrm{~mm}$ using the SEST telescope with an angular resolution of $23^{\prime \prime}$. They detected cold dust emission from G341.220-0.213 and
G341.217-0.237 and an extended halo linked to S 24, and estimated that the total mass in the region amounts to almost $5000 M_{\odot}$ (adopting a gas-to-dust ratio of 100 and a distance of $3.7 \mathrm{kpc})$.

Previous studies toward the region of S 24 have shown that star formation is active. Two spots of maser emission were detected. The first spot is a methanol and water maser emitter (Bayandina et al. 2012; Urquhart et al. 2013; Walsh et al. 2014) with peak velocities of between $\sim-50 \mathrm{~km} \mathrm{~s}^{-1}$ and $-38 \mathrm{~km} \mathrm{~s}^{-1}$ (Pestalozzi et al. 2005; Caswell et al. 2010; Voronkov et al. 2014; Walsh et al. 2014), coincident with G341.220-0.213. The second spot is also a methanol maser emitter (Vassilev et al. 2008; Bayandina et al. 2012) with peak velocities of between $\sim-70 \mathrm{~km} \mathrm{~s}^{-1}$ and $-16 \mathrm{~km} \mathrm{~s}^{-1}$ (Larionov \& Val'tts 2008; Voronkov et al. 2014) located at RA, Dec $(J 2000) \approx\left(16^{\mathrm{h}} 52^{\mathrm{m}} 15^{\mathrm{s}} .9\right.$, $-44^{\circ} 28^{\prime} 36^{\prime \prime}$.7), coincident with the IRDC SDC341.194-0.221. Although a number of EGO candidates have been identified in the S21-S24 complex (Cyganowski et al. 2008), none of them appear projected in the close environs of the IR bubble.

$\mathrm{S} 24$ is detected as a radio continuum source in the Molonglo Galactic Plane Survey at $843 \mathrm{MHz}$ (MGPSJ165220-442802, Murphy et al. 2007; synthesized beam $\left.=65^{\prime \prime} \times 45^{\prime \prime}\right)$ with an integrated flux density $S_{0.843}=0.92 \pm 0.04 \mathrm{Jy}$.

Bronfman et al. (1996) performed CS(2-1) line observations with an angular resolution of 50" using the SEST telescope. They observed the molecular line in one position toward IRAS16487-4423 $\left(\mathrm{RA}, \operatorname{Dec}(\mathrm{J} 2000)=\left(16^{\mathrm{h}} 52^{\mathrm{m}} 21.24^{\mathrm{s}}\right.\right.$, $\left.-44^{\circ} 28^{\prime} 2^{\prime \prime} .5\right)$ ) detecting CS emission with a velocity of $-43.4 \mathrm{~km} \mathrm{~s}^{-1}$, which indicates dense gas toward the IR source. Russeil \& Castets (2004) observed the $\mathrm{CO}(1-0)$ and $\mathrm{CO}(2-1)$ lines with an angular resolution of $42^{\prime \prime}$ and $21^{\prime \prime}$, respectively, toward a position located 4.3 to the southeast of the $S 24$ bubble, detecting molecular gas with velocities in the range $-40 \mathrm{~km} \mathrm{~s}^{-1}$ 
to $-37 \mathrm{~km} \mathrm{~s}^{-1}$. All velocities in this paper are given with reference to the LSR.

Circular galactic rotation models predict that gas with velocities of $-43.4 \mathrm{~km} \mathrm{~s}^{-1}$ lies at near and far kinematical distances of $3.6 \mathrm{kpc}$ and $12-13 \mathrm{kpc}$ (see, for example, Brand \& Blitz 1993). Similar distances can be derived from maser velocities. Following Henning et al. (2001), we adopt a distance of $3.7 \mathrm{kpc}$ for the S 24 region and its environment. Taking into account a velocity dispersion of $6 \mathrm{~km} \mathrm{~s}^{-1}$ for the interstellar gas, we adopt a distance uncertainty of $0.5 \mathrm{kpc}$.

Here, we present the very first molecular line and dust continuum study toward S 24 and its environs aimed at determining the distribution of the molecular gas and the dust linked to the bubble and the correlation between them, analyzing their physical conditions, masses, and ambient densities, and investigating the status of star formation in the region. The dense gas linked to this region makes it particularly interesting to investigate the physics and kinematics of HII regions and bubbles in dense media, allowing a better understanding of the evolution and star formation processes around these objects. Preliminary results were published in Cappa et al. (2013).

\section{Data sets}

\subsection{Molecular line observations}

The molecular gas characteristics were investigated by performing ${ }^{13} \mathrm{CO}(3-2)$ line observations (at $330.58797 \mathrm{GHz}$ ) of a region of $3 ! 2 \times 3.2$ during August 2009, and ${ }^{12} \mathrm{CO}(2-1),{ }^{13} \mathrm{CO}(2-1)$, and $\mathrm{C}^{18} \mathrm{O}(2-1)$ line observations of a region of $5^{\prime} \times 5^{\prime}$ obtained in October 2010, with the APEX telescope using the APEX-1 and APEX-2 receivers, whose system temperatures are $150 \mathrm{~K}$ and $300 \mathrm{~K}$, respectively.

The half-power beam-widths of the telescope are 30" (for the (2-1) lines) and $21^{\prime \prime}$ (for the (3-2) lines). The data were acquired with a FFT spectrometer, consisting of 4096 channels, with a total bandwidth of $1000 \mathrm{~km} \mathrm{~s}^{-1}$ and a velocity resolution of $0.33 \mathrm{~km} \mathrm{~s}^{-1}$. The region was observed in position-switching mode using the OTF technique with a space between dumps in the scanning direction of $9^{\prime \prime}$ for the $(2-1)$ transition, and in position-switching mode in the (3-2) transition. The off-source position free of CO emission was located at RA, Dec $(J 2000)=$ $\left(13^{\mathrm{h}} 33^{\mathrm{m}} 10.3^{\mathrm{s}},-62^{\circ} 2^{\prime} 41^{\prime \prime}\right)$.

Calibration was performed using Mars and X-TrA sources. Pointing was made twice during observations using X-TrA, oCeti, and VY-CMa. The intensity calibration has an uncertainty of $10 \%$.

The spectra were reduced using the Continuum and Line Analysis Single-dish Software (CLASS) of the Grenoble Image and Line Data Analysis Software (GILDAS) working group ${ }^{1}$. A linear baseline fitting was applied to the data. The rms noise of the profiles after baseline subtraction and calibration is $0.35 \mathrm{~K}$ for the (2-1) and (3-2) transitions. The observed line intensities are expressed as main-beam brightness temperatures $T_{\mathrm{mb}}$, by dividing the antenna temperature $T_{\mathrm{A}}$ by the main-beam efficiency $\eta_{\mathrm{mb}}$, equal to 0.72 for APEX-1 and 0.82 for APEX-2 (Vassilev et al. 2008). The Astronomical Image Processing System (AIPS) package and CLASS software were used to perform the analysis.

\footnotetext{
1 http://www.iram.fr/IRAMFR/PDB/class/class.html
}

\subsection{Dust continuum data}

To trace cold dust emission we used far-infrared (FIR) images from the Herschel Space Observatory ${ }^{2}$ and from the APEX Telescope Large Area Survey of the Galaxy (ATLASGAL, Schuller et al. 2009).

\subsubsection{Herschel data}

The archival data correspond to the Hi-GAL key program (HiGAL:Herschel Infrared GALactic plane survey, Molinari et al. 2010, OBSIDs: 1342204094 and 1342204095).

The data have been taken in parallel mode with the instruments PACS (Poglitsch et al. 2010) at 70 and $160 \mu \mathrm{m}$, and SPIRE (Griffin et al. 2010) at 250, 350, and $500 \mu \mathrm{m}$. The chosen tile covers a field of $2.2 \mathrm{sq}$. deg and is approximately centered at $(l, b)=\left(340^{\circ}, 0^{\circ}\right)$. The angular resolutions for the five photometric bands spans from $8^{\prime \prime}$ to $35^{\prime \prime} .2$ for $70 \mu \mathrm{m}$ to $500 \mu \mathrm{m}$. Herschel interactive processing environment (HIPE v12 ${ }^{3}$, Ott 2010) was used to reduce the data, with reduction scripts from standard processing. To combine the two obsids (scan and cross-scan) of PACS archive data, we used the HIPE implementation script of the map merger called Scanmorphos. This script starts from Level 1 frames and creates a map from the data. The obtained pixel sizes are $2^{\prime \prime} / \mathrm{px}$ to blue and $3^{\prime \prime} / \mathrm{px}$ to red.

The zero-level of the PACS and SPIRE archive data is unknown, which may cause problems, for example, to derive dust temperatures. The zero-level offsets of the SPIRE maps were corrected as described in Bernard et al. (2010). A proper zerolevel was set bearing in mind that Planck includes the same fraction of the sky as Hi-GAL data and taking into account that the two common photometric channels of Planck and SPIRE $(857 \mathrm{GHz} \sim 350 \mu \mathrm{m}$ and $545 \mathrm{GHz} \sim 550 \mu \mathrm{m})$ are calibrated with respect to FIRAS (Piat et al. 2002). We used the HIPE implementation script to run destriper gain corrections and to recalculate the absolute offset via cross-calibration with Planck-HFI data (HIPE v12, updated version by L. Conversi 2013).

To estimate fluxes, a Gaussian smoothing was applied to convolve all images at a low resolution of $500 \mu \mathrm{m}$ (a FWHM of $35^{\prime \prime} .2$ ).

Assuming that an extended source has a spectral index $\alpha_{\mathrm{s}}=$ 4 ( 2 for the blackbody emission plus 2 for the opacity of the dust), the obtained fluxes of each source were multiplied by $0.98755,0.98741$, and 0.96787 for 250,350 , and $500 \mu \mathrm{m}$, respectively, to convert from monochromatic intensities of point sources to monochromatic extended sources.

\subsubsection{ATLASGAL data}

This survey was performed at $870 \mu \mathrm{m}(345 \mathrm{GHz})$ with an angular resolution of 19.'2 (HPBW), using the Large Apex Bolometer Camera (LABOCA; Siringo et al. 2009). The camera operates with 295 pixels at the APEX 12-m submillimeter telescope.

\footnotetext{
2 Herschel is an ESA space observatory with science instruments provided by European-led Principal Investigator consortia and with important participation from NASA.

3 HIPE is a joint development by the Herschel Science Ground Segment Consortium, consisting of ESA, the NASA Herschel Science Center, and the HIFI, PACS and SPIRE consortia members, see http://herschel.esac.esa.int/HerschelPeople.shtml
} 


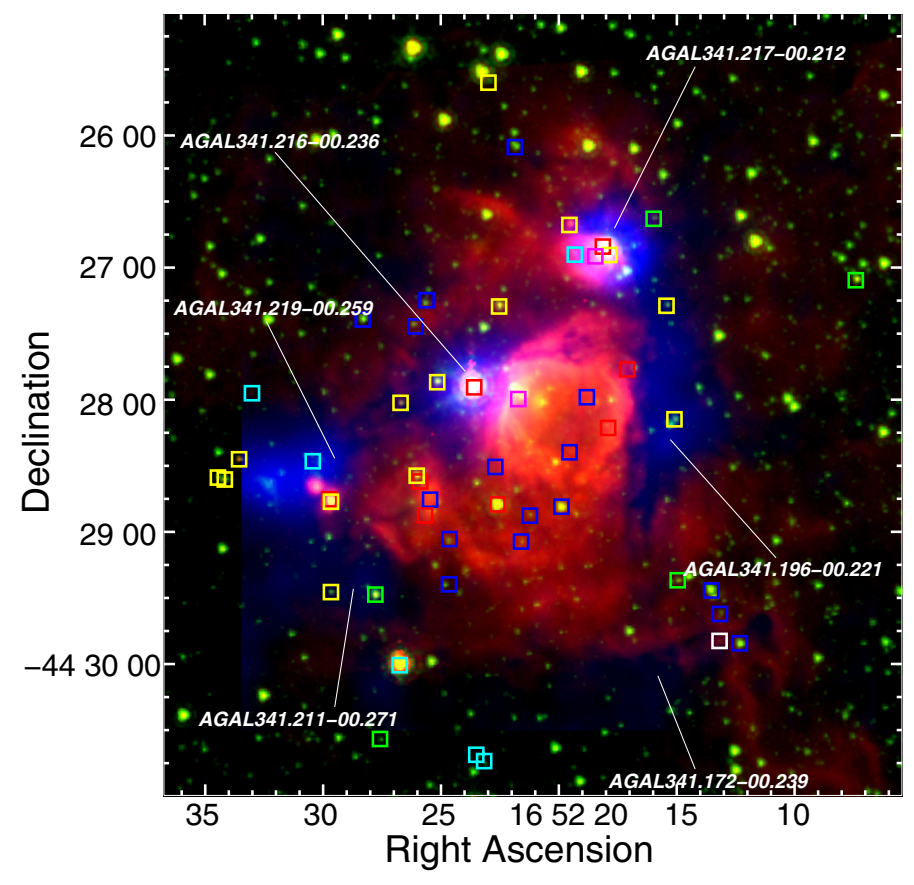

Fig. 2. Overlay of the IRAC emissions at $8 \mu \mathrm{m}$ (in red) and $3.6 \mu \mathrm{m}$ (in green) and the LABOCA emission at $870 \mu \mathrm{m}$ (in blue). Positions of identified YSOs are indicated with squares in different colors: Class I (red) and Class II (blue) sources from the WISE database, Class I (yellow), Class II (white), and Class I/II (green) sources from the Spitzer database, 2MASS sources (cyan), and MSX sources (magenta). Dust clumps from Contreras et al. (2013) are indicated.

\subsection{Complementary data}

The millimeter and submillimeter data were complemented with Spitzer images at 3.6, 4.5, and $8.0 \mu \mathrm{m}$ from the Galactic Legacy Infrared Mid-Plane Survey Extraordinaire (GLIMPSE; Benjamin et al. 2003), and at $24 \mu \mathrm{m}$ from the MIPS Inner Galactic Plane Survey (MIPSGAL; Carey et al. 2005). We used radio continuum images at $843 \mathrm{MHz}$ from the Molonglo Galactic Plane Survey (MGPS2, Murphy et al. 2007) with a synthesized beam of $65^{\prime \prime} \times 45^{\prime \prime}$, and at $1.4 \mathrm{GHz}$ from the Southern Galactic Plane Survey (Haverkorn et al. 2006; beam size = 1'.7).

To investigate the existence of YSO candidates projected onto the region, we analyzed infrared point sources from the Midcourse Space Experiment (MSX, Price et al. 2001), the Two Micron All Sky Survey (2MASS, Cutri et al. 2003), the Spitzer (Fazio et al. 2004), and the Wide-field Infrared Survey Explorer (WISE, Wright et al. 2010).

\section{YSOs projected onto the region}

As mentioned in Sect. 1, maser emission linked to star-forming regions has been detected in the region. Bearing this in mind, we decided to search for YSO candidates using the MSX, 2MASS, WISE, and Spitzer point source catalogs. To accomplish this, we analyzed the IR sources in a region of $3^{\prime}$ in radius centered at $\mathrm{RA}, \operatorname{Dec}(\mathrm{J} 2000)=\left(16^{\mathrm{h}} 52^{\mathrm{m}} 20^{\mathrm{s}},-44^{\circ} 28^{\prime} 00^{\prime \prime}\right)$. The results are included in Table 1, and their positions are shown in Fig. 2 with different symbols.

To identify YSO candidates in the MSX catalog, we followed the criteria of Lumsden et al. (2002), which take into account their loci in the $\left(\mathrm{F}_{21} / \mathrm{F}_{8}, \mathrm{~F}_{14} / \mathrm{F}_{12}\right)$ diagram. Two candidates were found, classified as massive YSOs (MYSO), one of them linked to G341.220-0.213 and the other one to the brightest section of the $\mathrm{S} 24$ bubble.
To search for YSO candidates in the 2MASS catalog, we have constructed an $\left[H-K_{\mathrm{S}}, J-H\right]$ diagram using only sources with photometric quality A or B. Six candidates were identified. The two upper panels of Fig. 3 show the 2MASS color-color (CC) and color-magnitude (CM) diagrams with the YSO candidates represented by red asterisks.

We have performed a search for candidates in the Spitzer database adopting the criteria by Allen et al. (2004), which allow distinguishing IR sources according to a class scheme: Class I are protostars surrounded by dusty infalling envelopes, and Class II are objects whose emission is dominated by accretion disks. Considering only sources detected in the four SpitzerIRAC bands, we investigated their evolutionary stage by analyzing their location in a color-color diagram (middle panel of Fig. 3) following the color criteria determined empirically by Allen et al. (2004). Class II candidates lie in the region $0 \lesssim[3.6]-[4.5] \lesssim 0.8$ and $0.4 \lesssim[5.8]-[8.0] \lesssim 1.1$, and their IR excess might be produced by accretion disks around the stellar objects. Sources outside the domain of Class II objects are identified as Class I candidate objects, and their IR excess might be due to circumstellar envelopes around the star. We identified 14 Class I candidates, 5 Class I/II candidates, and one Class II. The middle panel of Fig. 3 shows the position of the 20 Spitzer sources in the color-color [3.6]-[4.5] vs. [5.8]-[8.0] diagram. In this panel, the area delineated by thin lines corresponds to the locus of Class I objects, while the area within thick dashed lines corresponds to class II objects.

Finally, with the aim of identifying candidate YSOs using photometric data from the Wide-field Infrared Survey Explorer survey, which maps the whole sky in four bands centered at 3.4, 4.6, 12, and $22 \mu \mathrm{m}$, we used the criteria of Koenig et al. (2012), using [3.4]-[4.6] vs. [4.6]-[12] diagrams as follows: after removing contamination arising from background objects such as galaxies (very red in [4.6]-[12]), broad-line active galactic nuclei (of similar colors as YSOs, but distinctly fainter), and resolved PAH emission regions (redder than the majority of YSOs), we identified infrared excess sources by requiring that

$[3.4]-[4.6]-\sigma_{1}>0.25$

$[4.6]-[12.0]-\sigma_{2}>1.0$,

where [3.4], [4.6], and [12.0] are the WISE bands 1, 2, and 3, respectively, and $\sigma_{1}=\sigma([3.4]-[4.6])$ and $\sigma_{2}=\sigma([4.6]-[12.0])$ indicate the combined errors of [3.4]-[4.6] and [4.6]-[12.0] colors, added in quadrature. Class I sources are a subsample of this defined by

$$
\begin{aligned}
& {[3.4]-[4.6]>1.0} \\
& {[4.6]-[12.0]>2.0}
\end{aligned}
$$

The remaining sources are then Class II objects. Identified Class I and Class II candidates are shown in the bottom panel of Fig. 3. In this panel, the separation between the location of ClassI and Class II sources is indicated by the dashed lines.

As can be seen from Fig. 2, four candidates (one MYSO, one 2MASS source, one Spitzer Class I, and one WISE Class I) appear projected onto G341.220-0.213. This fact along with the presence of maser emission towards the region confirms that this is an active star-forming region. Three candidates coincide in position with the S 24 bubble, while only one WISE Class II candidate is projected onto G341.217-0.237. Most of the candidates appear to the east of the IRDC SDC341.193-0.221 and are projected onto emission at $8 \mu \mathrm{m}$. Finally, two Spitzer candidates coincide with the IRDC SDC341.194-0.221, and another three candidates appear projected onto the borders of G341.210-0.252. 
Table 1. YSO candidates projected onto S 24 and its environs.

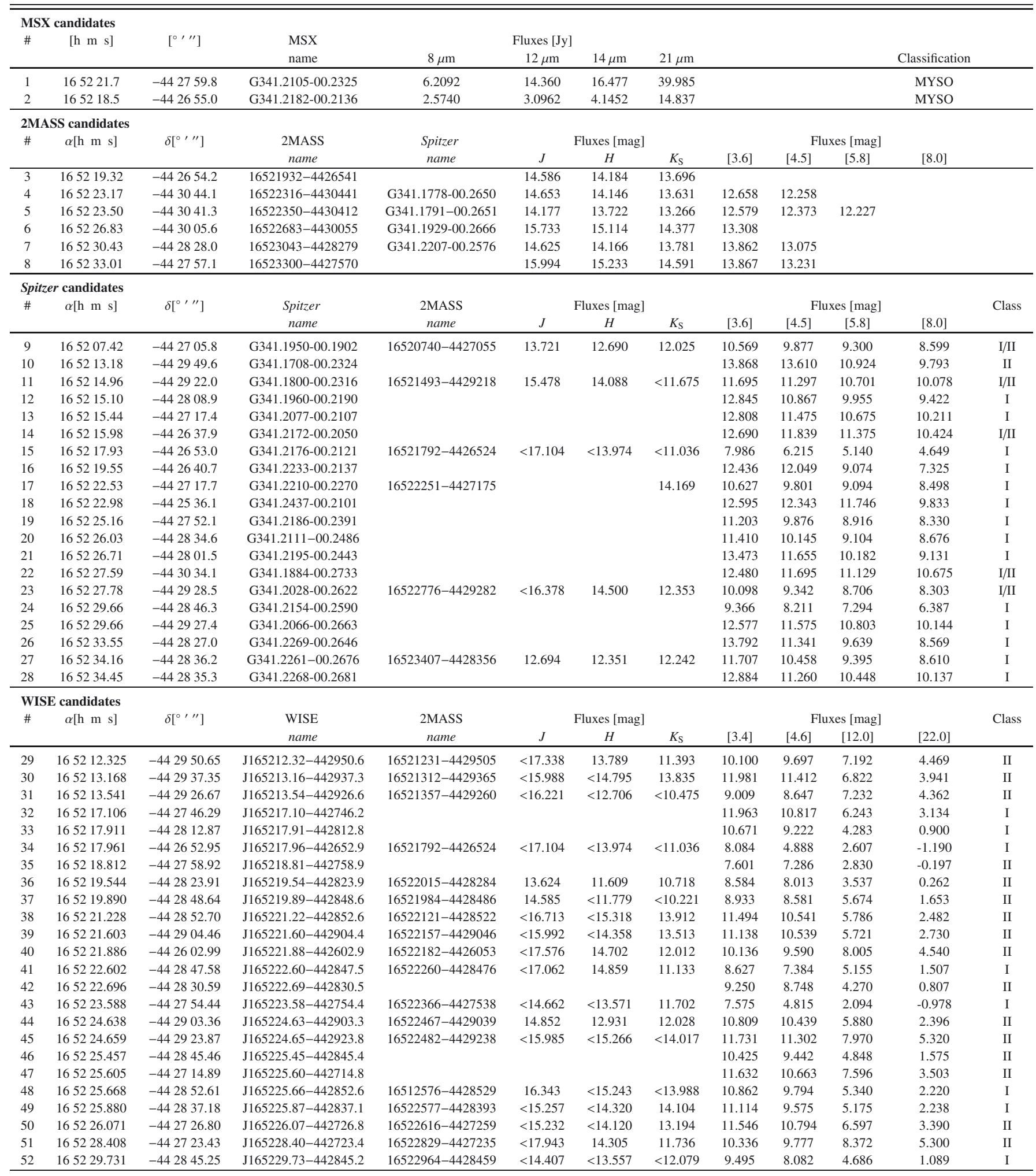

It is clear from Table 1 that there is little correspondence among 2MASS, Spitzer, and WISE candidate YSOs (coincidences: \#24 with \#52; and \#2 with \#15 and \#34). In spite of the similar wavelengths of two of the bands and in the different angular resolution of the Spitzer and WISE catalogs, an analysis of the coincidence of Spitzer and WISE YSOs identified in Table 1 shows that $33 \%$ of the WISE and Spitzer sources do not have a counterpart in the respective other catalog. Additionally, 35\% of the WISE and Spitzer sources coincide with Spitzer and WISE sources, respectively, which are undetected in at least one band and cannot be properly classified. For the remaining 17\%, the Spitzer and WISE sources coincide, but their counterparts are not classified as Class I or II. 

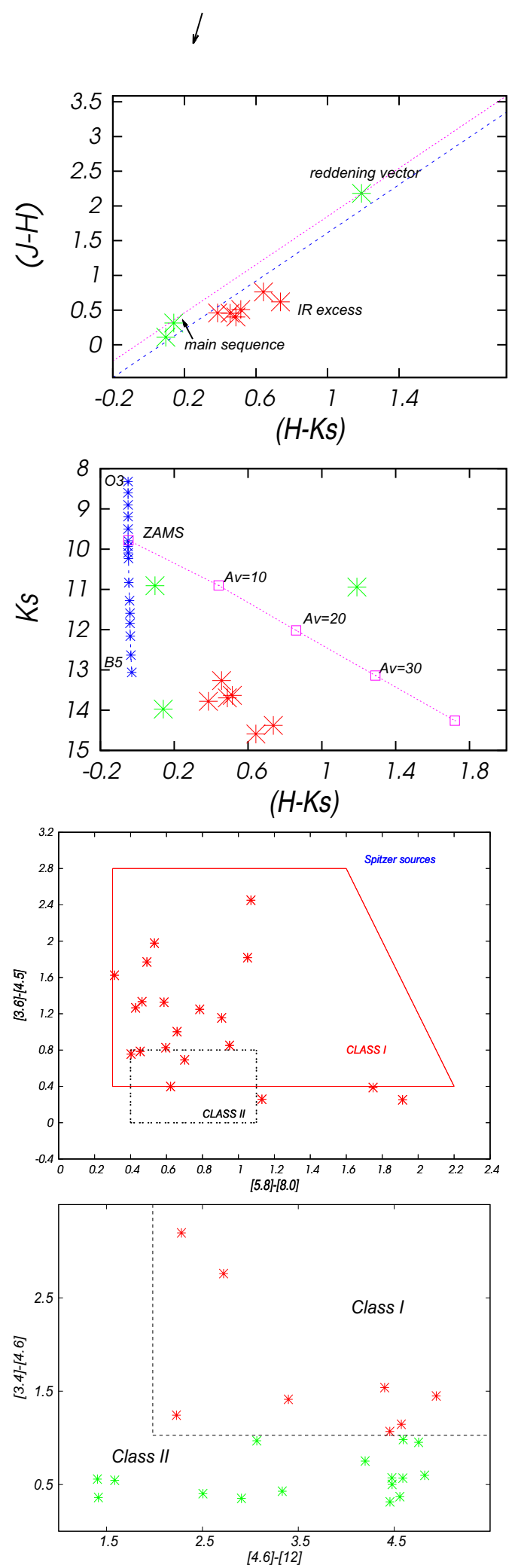

Fig. 3. Upper panels: $\left[H-K_{\mathrm{s}}, J-H\right]$ and $\left[H-K_{\mathrm{s}}, K_{\mathrm{S}}\right]$ diagrams of the 2MASS YSO candidates indicated by red crosses. Green asterisks correspond to main sequence stars projected onto the $\mathrm{S} 24$ bubble (see Sect. 7.2). The dashed lines show the reddening vectors corresponding to M0 III and B2 V stars in the CC-diagram, and to B2 V stars in the $\mathrm{CM}$-diagram. The squares are placed at intervals of ten magnitudes of visual extinction. Middle panel: location of YSO candidates from the Spitzer database. Crosses represent Class I and II sources. Bottom panel: YSO candidates from the WISE database. Class I and II sources are indicated in red and green, respectively.

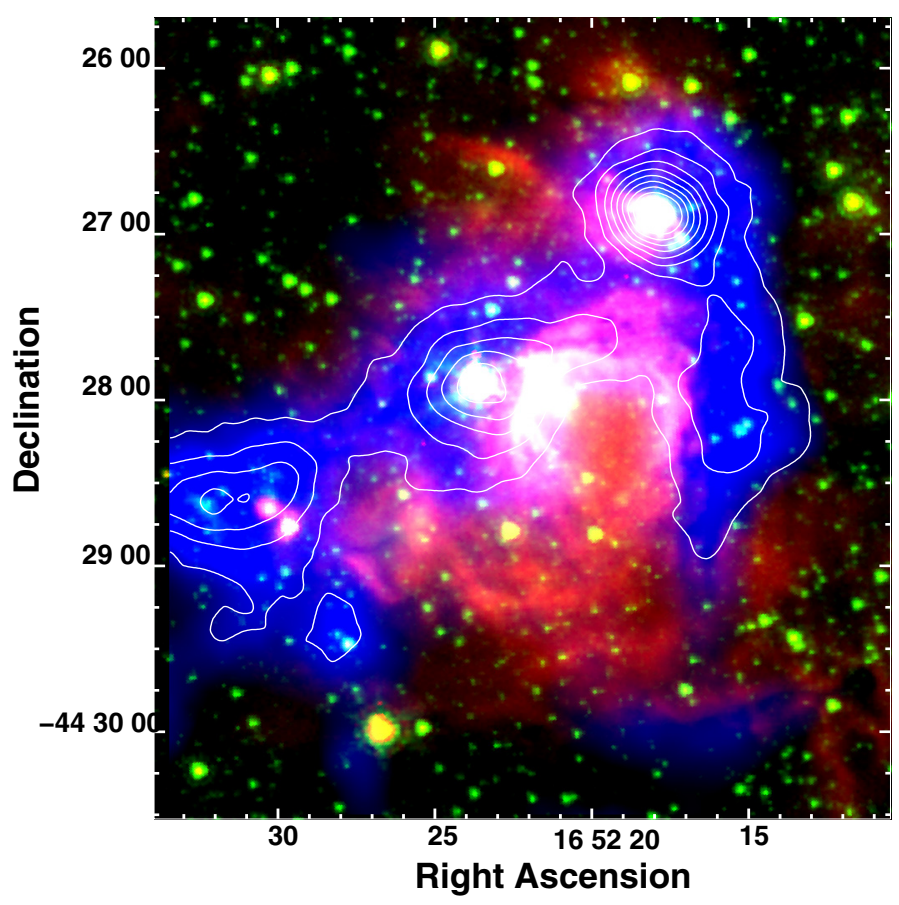

Fig. 4. Composite image of the IRAC emission at 4.5 (in green) and $8.0 \mu \mathrm{m}$ (in red), and the Herschel emission at $250 \mu \mathrm{m}$ (in blue). Contours show the emission at $870 \mu \mathrm{m}$ and correspond to $0.5 \mathrm{MJy} \mathrm{sr}^{-1}$ to $4.0 \mathrm{MJy} \mathrm{sr}^{-1}$ in steps of $0.5 \mathrm{MJy} \mathrm{sr}^{-1}$.

A more detailed analysis is performed in connection to the molecular dust and cold dust in the next sections.

\section{Distribution of the interstellar dust}

The distribution of the interstellar dust was investigated using data at different wavelengths in the IR.

Figure 2 shows a superposition of the IRAC $8 \mu \mathrm{m}$ (in red) and $3.6 \mu \mathrm{m}$ (in green) emissions, and the ATLASGAL $870 \mu \mathrm{m}$ emission (in blue). Emission at $870 \mu \mathrm{m}$ reveals the presence of cold dust. This emission approximately encircles the S24 bubble. The dust clumps identified at this wavelength by Contreras et al. (2013) are indicated. AGAL341.21600.236 and AGAL341.217-00.212 are bright, coincide with G341.217-0.237 and G341.220-0.213, respectively, and correlate with the emission at $1.2 \mathrm{~mm}$ detected by Henning et al. (2001). Weaker emission at $870 \mu \mathrm{m}$ (AGAL SDC341.19600.221) coincides in position with the IRDC SDC341.1940.221 placed to the west of the bubble following its sharp eastern border, and is evident from the $8 \mu \mathrm{m}$ emission in Fig. 2. Additionally, two cold dust clumps are located to the south and southeast of S 24, at RA, Dec(J2000) = $\left(16^{\mathrm{h}} 52^{\mathrm{m}} 19.6^{\mathrm{s}},-44^{\circ} 30^{\prime} 38^{\prime \prime} .5\right)$ (AGAL SDC341.172-00.239), and $\mathrm{RA}, \operatorname{Dec}(\mathrm{J} 2000)=\left(16^{\mathrm{h}} 52^{\mathrm{m}} 31.71^{\mathrm{s}},-44^{\circ} 29^{\prime} 26^{\prime \prime} .1\right)(\mathrm{AGAL}$ SDC341.211-00.271). AGAL SDC341.219-00.259, located at $\mathrm{RA}, \operatorname{Dec}(\mathrm{J} 2000)=\left(16^{\mathrm{h}} 52^{\mathrm{m}} 32^{\mathrm{s}},-44^{\circ} 28^{\prime} 30^{\prime \prime}\right)$, coincides with a section of the IRDC SDC341.232-0.268 and is being analyzed by Vasquez et al. (in prep.). G341.210-0.252 does not coincide with emission at $870 \mu \mathrm{m}$.

Figure 4 displays an overlay of the emission at $870 \mu \mathrm{m}$ (in contours) and the emissions at 4.5 (in green) and $8.0 \mu \mathrm{m}$ (in red) from Spitzer and $250 \mu \mathrm{m}$ (in blue) from Herschel. Dust clumps identified at $870 \mu \mathrm{m}$ have counterparts at $250 \mu \mathrm{m}$, although the emission at $250 \mu \mathrm{m}$, which also shows the distribution of cold dust, appears more extended. The brightest dust 

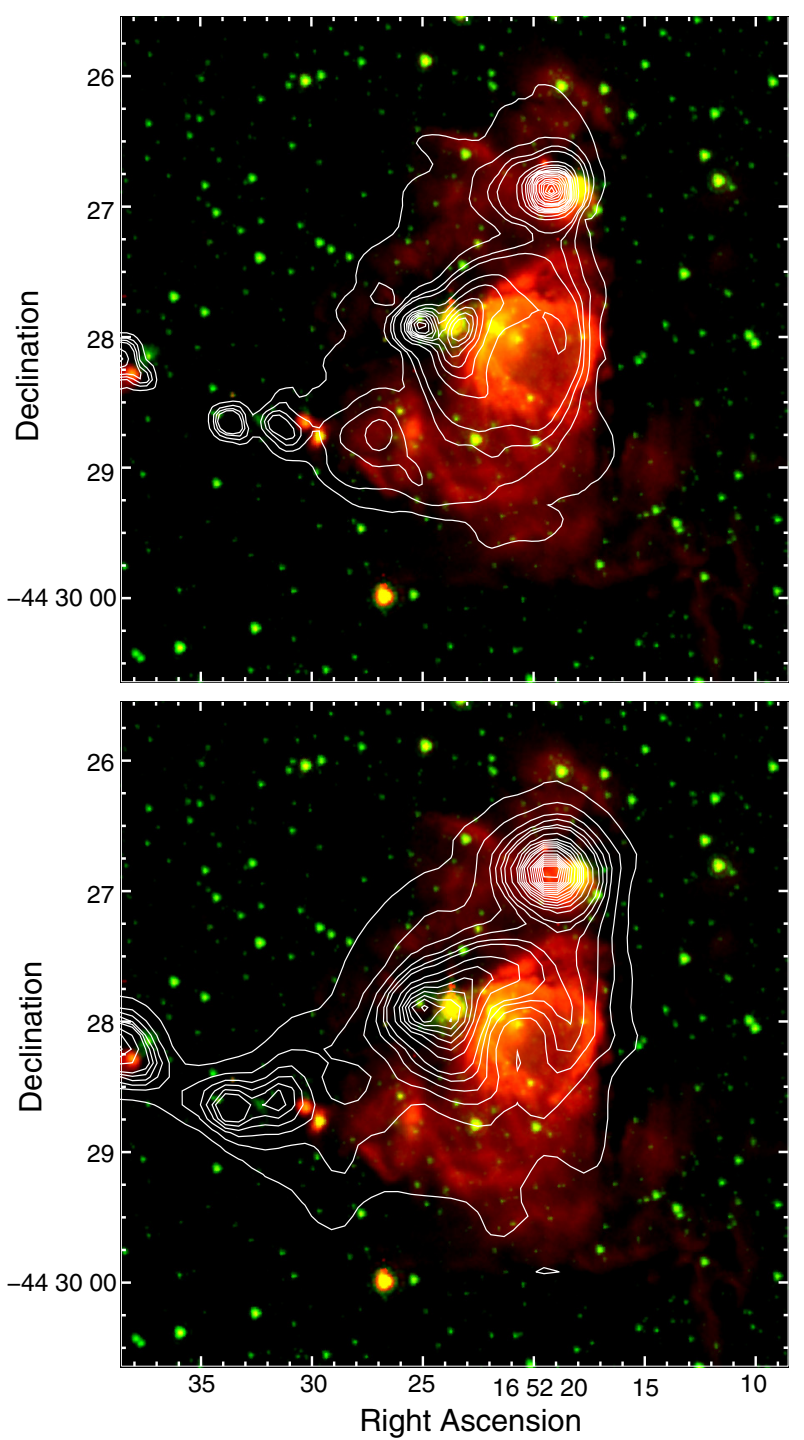

Fig. 5. Upper panel: overlay of the PACS emission at $70 \mu \mathrm{m}$ (contours) and the IRAC $8 \mu \mathrm{m}$ emission (colorscale). Contours are 0.3 to $0.9 \mathrm{Jy} \mathrm{px}^{-1}$ in steps of $0.2 \mathrm{Jy} \mathrm{px}^{-1} ; 2$ to $7 \mathrm{Jy} \mathrm{px}^{-1}$ in steps of $1 \mathrm{Jy} \mathrm{px}^{-1}$; and $9,11,13$ to $33 \mathrm{Jy} \mathrm{px}^{-1}$ in steps of $4 \mathrm{Jy} \mathrm{px}^{-1}$. Bottom panel: overlay of the PACS emission at $160 \mu \mathrm{m}$ (contours) and the IRAC $8 \mu \mathrm{m}$ emission (colorscale). Contours are 2 to $7 \mathrm{Jy} \mathrm{px}^{-1}$ in steps of $1 \mathrm{Jy} \mathrm{px}^{-1}$; 10 to $25 \mathrm{Jy} \mathrm{px}^{-1}$ in steps of $3 \mathrm{Jy} \mathrm{px}^{-1}$; and 30 to $65 \mathrm{Jy} \mathrm{px}^{-1}$ in steps of $5 \mathrm{Jy} \mathrm{px}^{-1}$.

clumps at $250 \mu \mathrm{m}$ coincide with G341.220-0.213 and G341.2170.237 and are also detected at 350 and $500 \mu \mathrm{m}$ (the images are not shown here). The S 24 bubble appears clearly surrounded to the east and north by SPIRE emission. Herschel emission at these wavelengths is also projected onto the IRDC identified to the west of S 24, which can be defined as a Herschel bright IRDC (Peretto et al. 2010). Faint extended emission at $250 \mu \mathrm{m}$ also coincides with the IRDC SDC341.171-0.241, bordering the weak halo of $8 \mu \mathrm{m}$ emission, and near RA, Dec $(J 2000)=\left(16^{\mathrm{h}} 52^{\mathrm{m}} 28^{\mathrm{s}}\right.$, $\left.-44^{\circ} 29^{\prime} 30^{\prime \prime}\right)$, in coincidence with a Spitzer point source identified at $4.5 \mu \mathrm{m}$. In summary, the correlation between 250 and $870 \mu \mathrm{m}$ is excellent.

The PACS emission at 70 and $160 \mu \mathrm{m}$ differs significantly from the emission at longer wavelengths (Fig. 5). The brightest source at these wavelengths coincides with G341.220-0.213. G341.217-0.237 also emits at 70 and $160 \mu \mathrm{m}$, while a bright source is also detected to the east of G341.217-0.237. Faint

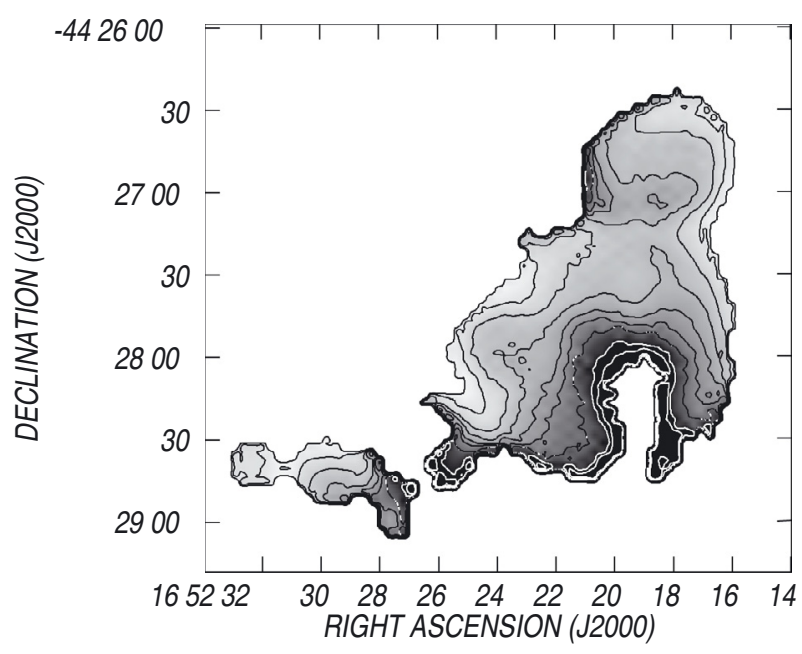

Fig. 6. Dust temperature map derived from the Herschel emission at 70 and $160 \mu \mathrm{m}$. The grayscale extends from 20 to $50 \mathrm{~K}$. Contour levels correspond to 20 to $32 \mathrm{~K}$ in steps of $3 \mathrm{~K}, 35,40,50$, and $60 \mathrm{~K}$. The darkest regions indicate the highest dust temperatures.

emission correlates with the S 24 bubble, mainly at $70 \mu \mathrm{m}$. This emission delineates the sharp western border of the bubble. The IRDC SDC 341.194-0.221 cannot be identified at either $70 \mu \mathrm{m}$ or $160 \mu \mathrm{m}$. Emission at $70 \mu \mathrm{m}$ is also projected onto G341.210-0.252, located at $16^{\mathrm{h}} 52^{\mathrm{m}} 26.5^{\mathrm{s}},-44^{\circ} 28^{\prime} 50^{\prime \prime}$, but is lacking at $160 \mu \mathrm{m}$ and higher wavelengths. The presence of emission at $70 \mu \mathrm{m}$ and its absence at $160 \mu \mathrm{m}$ suggests the existence of dust at a higher temperature.

Dust temperatures $T_{\text {dust }}$ for the region using the PACS images at $70 \mu \mathrm{m}$ and $160 \mu \mathrm{m}$ were estimated by convolving the image at $70 \mu \mathrm{m}$ down to the angular resolution at $160 \mu \mathrm{m}$ by applying color corrections, subtracting a background local to the S 24 bubble, and assuming that the emission is optically thin.

The color-temperature map was constructed as the inverse function of the ratio map of Herschel $70 \mu \mathrm{m}$ and $160 \mu \mathrm{m}$ colorand-background-corrected maps, that is, $T_{\text {dust }}=f(T)^{-1}$ (see details in Preibisch et al. 2012; Ohlendorf et al. 2013). In the optically thin thermal dust emission regime $f(T)$ has the following parametric form:

$f(T)=\frac{S_{\nu}(v=70 \mu \mathrm{m})}{S_{v}(v=160 \mu \mathrm{m})}=\frac{B_{v}(70 \mu \mathrm{m}, T)}{B_{v}(160 \mu \mathrm{m}, T)}\left(\frac{70}{160}\right)^{\beta_{\mathrm{d}}}$,

where $B_{v}(v, T)$ is the blackbody Planck function and $\beta_{\mathrm{d}}$ corresponds to the spectral index of the thermal dust emission. The pixel-to-pixel temperature was calculated assuming $\beta_{\mathrm{d}}=2$. This is a typical value adopted for irradiated regions (see Preibisch et al. 2012).

The obtained dust temperature map is shown in Fig. 6. We were able to estimate dust temperatures for G341.220-0.213, G341.217-0.237, G341.210-0.252, and for the S 24 bubble. Dust temperatures using this method were almost impossible to obtain for the IRDCs due to their low emission at $70 \mu \mathrm{m}$. Values for G341.220-0.213 are in the range 26-30 K, while for G341.2170.237 temperatures are about $29 \mathrm{~K}$. The image reveals a clear gradient in dust temperatures (from 26 to $60 \mathrm{~K}$ ) for the $\mathrm{S} 24$ bubble: the higher the temperature, the closer to the center of the bubble. This is compatible with ionizing sources that are linked to S 24. Dust temperatures for S 24 are compatible with values derived by Watson et al. (2010) for the interior of IR dust bubbles based on images at 24 and $70 \mu \mathrm{m}$ from MIPS and by 


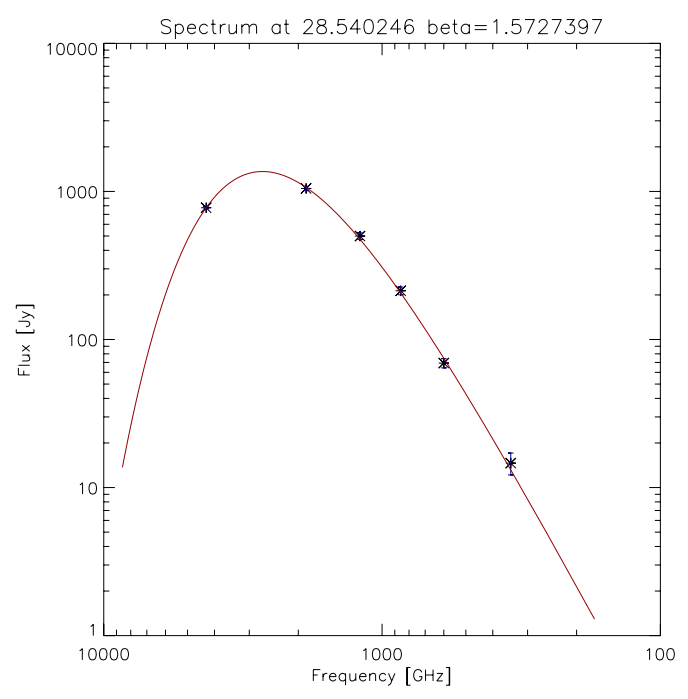

Fig. 7. SED for G341.220-0.213 obtained using Herschel and ATLASGAL fluxes.

Anderson et al. (2012) for HII regions using Herschel data. Dust temperatures for G341.217-0.237 are about $28 \mathrm{~K}$.

We also estimated $T_{\text {dust }}$ for G341.220-0.213 from the whole set of Herschel data and the LABOCA image by fitting a Planck function. Fluxes at 70, 160, 250, 350, and $500 \mu \mathrm{m}$ were calculated with HIPE using rectangular sky aperture photometry and convolving all images down to the angular resolution at $500 \mu \mathrm{m}$. For each aperture we measured the average surface brightness with an uncertainty derived from the standard deviation of the surface brightness within each aperture and the flux calibration uncertainty. Derived values are 776.0, 1047.2, 499.5, 213.3, and $69.3 \mathrm{Jy}$ at $70,160,250,350$, and $500 \mu \mathrm{m}$, respectively. Figure 7 shows the spectral energy distribution (SED), which also includes the flux density at $870 \mu \mathrm{m}$ obtained by Contreras et al. $(2013 ; 14.63 \pm 2.47 \mathrm{Jy})$, and the best fit (obtained using a graybody), which corresponds to $T_{\text {dust }}=28 \pm 1 \mathrm{~K}$. Dust temperature for G341.217-0.237 from an SED is difficult to determine. As a result of the poor angular resolution of the Herschel images at 350 and $500 \mu \mathrm{m}$, fluxes estimated from these data include part of S 24, which can give a false impression of the dust temperature.

Dust masses can be estimated from the expression (Hildebrand 1983)

$M_{\text {dust }}=\frac{S_{870} d^{2}}{\kappa_{870} B_{870}\left(T_{\text {dust }}\right)}$,

where $S_{870}$ is the flux density at $870 \mu \mathrm{m}, d=3.7 \pm 0.5 \mathrm{kpc}$, $\kappa_{870}=1.0 \mathrm{~cm}^{2} / \mathrm{gr}$ is the dust opacity per unit mass (Ossenkopf $\&$ Henning 1994), and $B_{870}\left(T_{\text {dust }}\right)$ is the Planck function for a temperature $T_{\text {dust }}$.

Two processes may contribute to the emission at this wavelength in addition to the thermal emission from cold dust: molecular emission from the $\mathrm{CO}(3-2)$ line and free-free emission from ionized gas. The continuum emission contribution at $345 \mathrm{GHz}$ due to ionized gas was estimated from the radio continuum image at $1.4 \mathrm{GHz}$ considering that the radio emission is thermal (see Sect. 5) using the expression $S_{345}=(345 / 1.4)^{-0.1} S_{1.4}$. Adopting $S_{1.4}=1.2 \mathrm{Jy}$ (see Sect. 5), this contribution amounts to about $1 \%$ of the emission at $870 \mu \mathrm{m}$. To estimate the contribution from $\mathrm{CO}(3-2)$, we took into account the intensity in the ${ }^{13} \mathrm{CO}(3-2)$ line. This mechanism contributes less than $1 \%$. Thus, the contribution by both mechanisms is within calibration uncertainties.

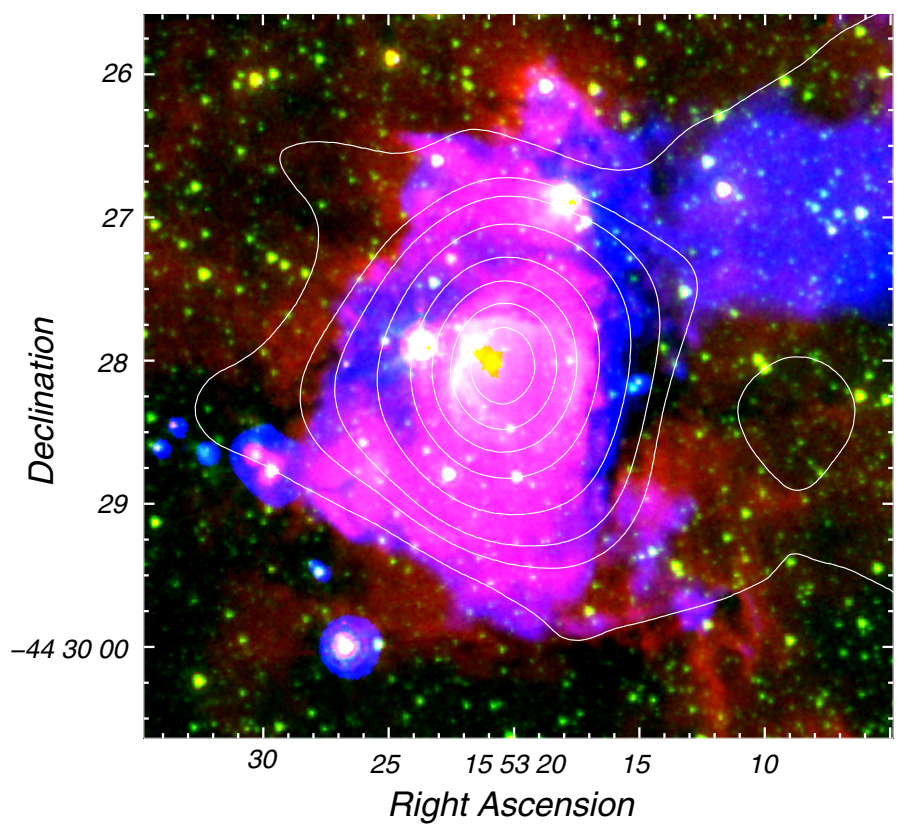

Fig. 8. Overlay of the radio continuum emission at $843 \mathrm{MHz}$ (contours), the IRAC emission at $8 \mu \mathrm{m}$ (in red), the MIPSGAL emission at $24 \mu \mathrm{m}$ (in blue), and the IRAC emission at $3.6 \mu \mathrm{m}$ (in green). Contours correspond to 10,30 , and $50 \mathrm{mJy}^{\text {beam }}{ }^{-1}$, and from 100 to $500 \mathrm{mJy}_{\text {beam }}^{-1}$ in steps of $100 \mathrm{mJy}^{\text {beam }}{ }^{-1}$.

Adopting $T_{\text {dust }}=28 \pm 1 \mathrm{~K}$ for G341.220-0.213, and a flux $S_{870}=14.63 \pm 2.47$ Jy derived by Contreras et al. (2013) for AGAL341.217-00.212, a dust mass $M_{\text {dust } 1}=12.9 \pm 6.3 M_{\odot}$ can be estimated for G341.220-0.213. Assuming the same dust temperature for G341.217-0.237and a flux $S_{870}=25.55 \pm$ 4.11 Jy (for AGAL341.216-00.236), we estimate $M_{\text {dust2 }}=$ $22.4 \pm 11.0 M_{\odot}$. Finally, for the IRDC to the west of the bubble, we adopt temperatures in the range 10-20 K (see Peretto et al. 2010), which, along with a flux $S_{870}=12.43 \pm 2.14 \mathrm{Jy}$ for AGAL341.196-00.221, results in $M_{\text {dust-irdc }}=17-57 M_{\odot}$. Uncertainties in these values are about $50 \%$. Adopting a gas-todust ratio of 100, gas masses are $1300 M_{\odot}$ for G341.220-0.213, $2300 M_{\odot}$ for G341.217-0.237, and 1700-5700 $M_{\odot}$ for the IRDC.

\section{5. lonized gas}

Figure 8 displays an overlay of the radio continuum image at $843 \mathrm{MHz}$ (in contours) and the emissions at $8 \mu \mathrm{m}$ and $24 \mu \mathrm{m}$ (in colorscale). The image at $843 \mathrm{MHz}$ reveals radio emission coincident with the sources, and in particular with the S 24 bubble. The emission at $1.4 \mathrm{GHz}$ (not shown here) correlates with that at the lower frequency. We derived a flux density $S_{1.4}=1.2 \mathrm{Jy}$. Taking into account a flux density $S_{0.843}=0.92 \mathrm{Jy}$, a spectral index $\alpha=0.5\left(S_{v} \propto v^{\alpha}\right)$ can be estimated from the emission at both frequencies. The $\alpha$-value indicates that at least at $843 \mathrm{MHz}$, the radio emission is optically thick, and that the source is thermal in nature.

Figure 8 also shows that S 24, as well as G341.220-0.213, G341.217-0.237, and G341.210-0.252 emit at $24 \mu \mathrm{m}$, indicating the presence of warm dust linked to the sources, and consequently, the existence of excitation sources inside them. Emission at this wavelength also coincides with the PDR, as revealed by the emission at $8 \mu \mathrm{m}$. In fact, the emission at $24 \mu \mathrm{m}$ from HII regions has two components: emission from very small grains out of thermal equilibrium, detected inside the HII region, 


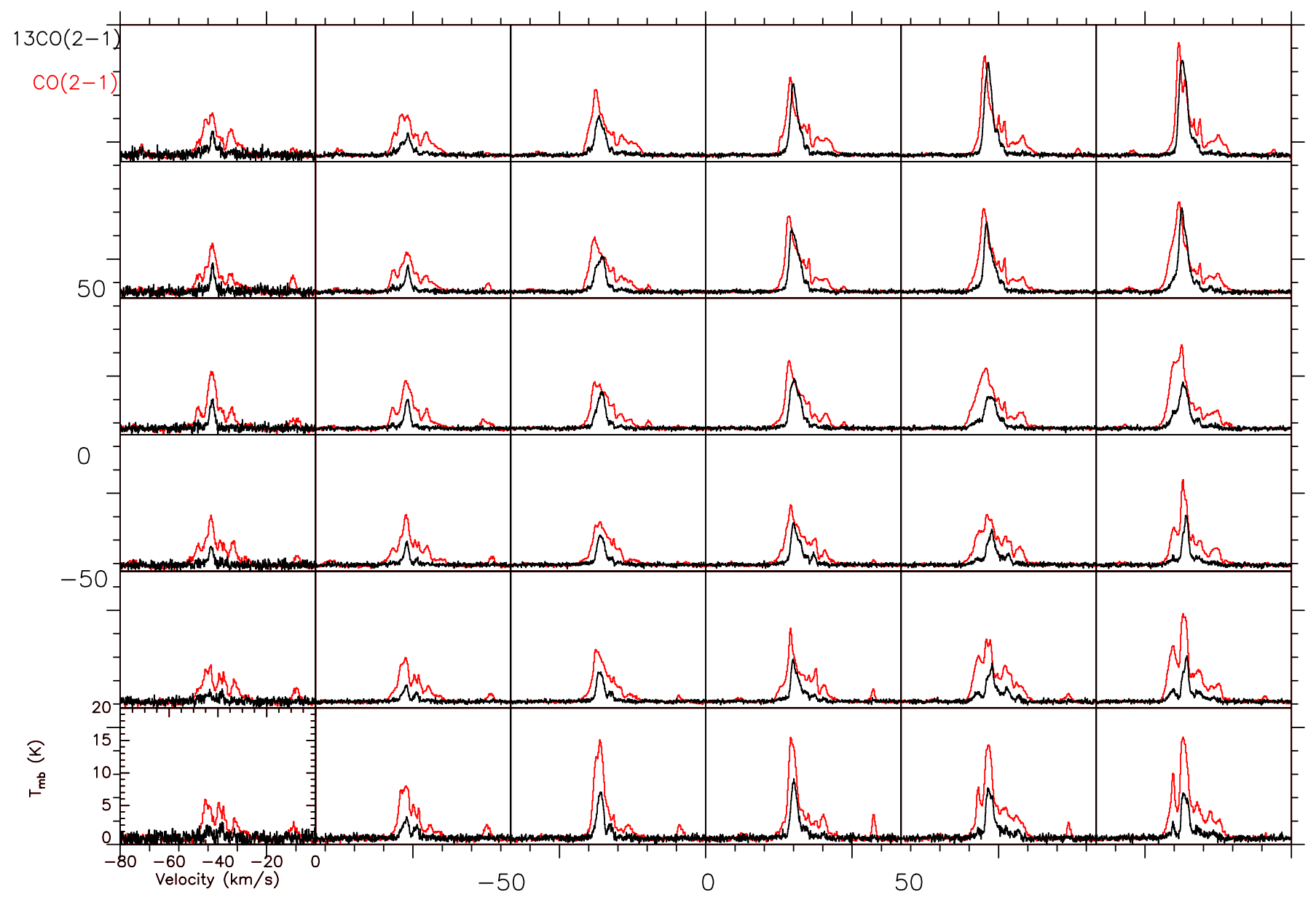

Fig. 9. Dec vs. RA map showing ${ }^{12} \mathrm{CO}(2-1)$ (in red) and ${ }^{13} \mathrm{CO}(2-1)$ (in black) profiles observed in a $5^{\prime} \times 5^{\prime}$ region centered in the $\mathrm{S} 24$ bubble. Each profile shows $T_{\mathrm{mb}}$ in the interval from $-1 \mathrm{~K}$ to $20 \mathrm{~K}$ vs. LSR velocity in the range from $-80 \mathrm{~km} \mathrm{~s}^{-1}$ to $0 \mathrm{~km} \mathrm{~s}^{-1}$. The $(0,0)$ position corresponds to RA, Dec $(\mathrm{J} 2000)=\left(16^{\mathrm{h}} 52^{\mathrm{m}} 20^{\mathrm{s}},-44^{\circ} 27^{\prime} 30^{\prime \prime}\right)$. Note that RA inceases towards the right.

and thermal emission from the PDR from grains in thermal equilibrium (Anderson et al. 2012).

Lower limits for the electron density $n_{\mathrm{e}}$ and the ionized mass $M_{i}$ can be derived from the image at $843 \mathrm{MHz}$. For an estimated flux density of $0.92 \mathrm{Jy}$, using the classical expressions by (Mezger \& Henderson 1967), a typical electron temperature of $10^{4} \mathrm{~K}$, and bearing in mind a $\mathrm{He}$ abundance of $10 \%$ by particle number and that He is single ionized, we derived $n_{\mathrm{e}}>200 \mathrm{~cm}^{-3}$, and $M_{\mathrm{i}}>22 M_{\odot}$. We note that similar results can be obtained using the emission at $1.4 \mathrm{GHz}$. Clearly, the fact that the emission at $1.4 \mathrm{GHz}$ is optically thick also can not be ruled out. Bearing in mind that the emission at $8 \mu \mathrm{m}$ due to PAH in the photodissociation region marks the boundary of the ionized region, we measured a radius of $0.33 \mathrm{pc}$ for the HII region.

Although these results can not be used to determine either the ionized mass or the electron density, they reveal the existence of an HII region.

\section{Distribution of the molecular gas}

Figure 9 displays the $\mathrm{CO}(2-1)$ and ${ }^{13} \mathrm{CO}(2-1)$ spectra obtained for the region under study. The spatial separation between these profiles is $30^{\prime \prime}$. Relative coordinates are expressed in arcseconds, referred to RA, Dec $(\mathrm{J} 2000)=\left(16^{\mathrm{h}} 52^{\mathrm{m}} 20^{\mathrm{s}},-44^{\circ} 27^{\prime} 30^{\prime \prime}\right)$. The individual spectra show that the molecular emission appears concentrated between $\sim-55 \mathrm{~km} \mathrm{~s}^{-1}$ and $-20 \mathrm{~km} \mathrm{~s}^{-1}$, where a number of velocity components are present, and an isolated component near $-10 \mathrm{~km} \mathrm{~s}^{-1}$ (which is not going to be taken into account). The $\mathrm{CO}(2-1) /{ }^{13} \mathrm{CO}(2-1)$ line ratios are close to $1-1.5$ in the central and northeastern parts of the figure, suggesting that the gas is optically thick at ${ }^{13} \mathrm{CO}(2-1)$ where S $24, \mathrm{G} 341.220$ 0.213 , and G341.217-0.237 are located.

The CO spectra of Fig. 10 illustrate the general behaviour of the molecular gas towards the entire region. There, we show the $\mathrm{CO}(2-1),{ }^{13} \mathrm{CO}(2-1)$, and $\mathrm{C}^{18} \mathrm{O}(2-1)$ spectra averaged within the observed region. At least three velocity components can be identified in the line of sight within the velocity interval from $-55 \mathrm{~km} \mathrm{~s}^{-1}$ to $-20 \mathrm{~km} \mathrm{~s}^{-1}$. The bulk of the molecular emission is detected in the three molecular lines and peaks at $\simeq-45 \mathrm{~km} \mathrm{~s}^{-1}$, while fainter gas components are present at $\simeq-38 \mathrm{~km} \mathrm{~s}^{-1}$ and $-30 \mathrm{~km} \mathrm{~s}^{-1}$. The faintest component is present near $-10 \mathrm{~km} \mathrm{~s}^{-1}$ in the ${ }^{12} \mathrm{CO}(2-1)$ line only. The velocity components peaking at $-38 \mathrm{~km} \mathrm{~s}^{-1}$ and $-30 \mathrm{~km} \mathrm{~s}^{-1}$ can be barely identified in the ${ }^{13} \mathrm{CO}(2-1)$ spectrum and are absent in the $\mathrm{C}^{18} \mathrm{O}(2-1)$ profile. Gas components having the same velocity can be also identified in the averaged $J=3-2$ profile (not shown here).

Circular galactic rotation models (e.g. Brand \& Blitz 1993) predict near and far kinematical distances in the range $3.2-3.9 \mathrm{kpc}$ and $12-13 \mathrm{kpc}$, respectively, for the gas component at $-45 \mathrm{~km} \mathrm{~s}^{-1}$, while near and far kinematical distances in the range $2.4-3.5$ and $12-14 \mathrm{kpc}$, respectively, were obtained for gas in the components at $-38 \mathrm{~km} \mathrm{~s}^{-1}$ and $-30 \mathrm{~km} \mathrm{~s}^{-1}$. Particularly for the component at $-45 \mathrm{~km} \mathrm{~s}^{-1}$, near kinematical 


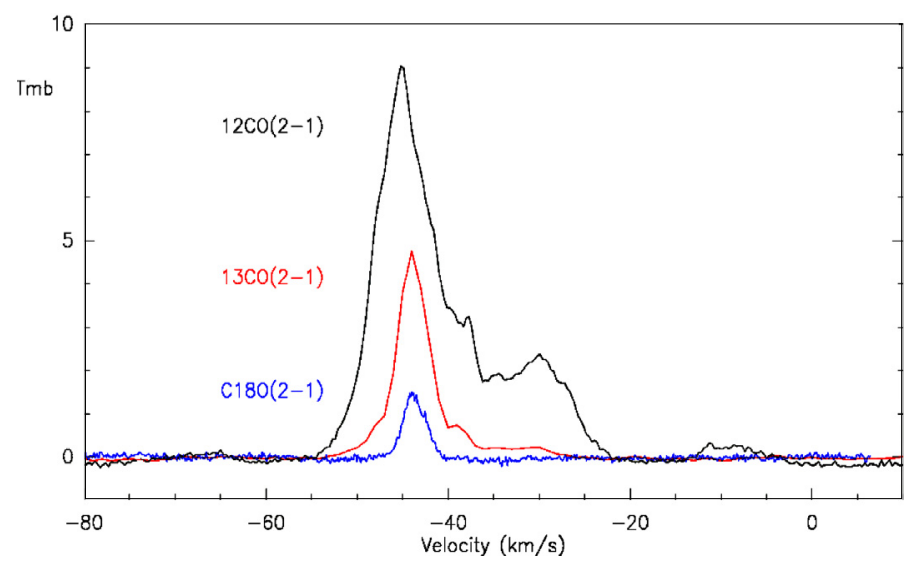

Fig. 10. $\mathrm{CO}(2-1)$ (in black), ${ }^{13} \mathrm{CO}(2-1)$ (in red), and $\mathrm{C}^{18} \mathrm{O}(2-1)$ (in blue) spectra obtained by averaging the observed spectra in the region of S 24. Intensity is expressed as main-beam brightness-temperature.

distances are compatible with previously adopted values for the region of S 24 .

To illustrate the spatial distribution of the molecular gas we show in Fig. 11 the emission of the ${ }^{13} \mathrm{CO}(2-1)$ in the interval $-49.9 \mathrm{~km} \mathrm{~s}^{-1}$ to $-40.5 \mathrm{~km} \mathrm{~s}^{-1}$, in steps of $1.05 \mathrm{~km} \mathrm{~s}^{-1}$ (in grayscale) superimpossed to the emission at $8 \mu \mathrm{m}$ (in contours) for comparison. Most of the molecular emission has velocities in the interval from $-46.8 \mathrm{~km} \mathrm{~s}^{-1}$ to $-41.5 \mathrm{~km} \mathrm{~s}^{-1}$.

As regards the $\mathrm{S} 24$ bubble, $\mathrm{CO}$ emission encircles the eastern and northern sections at $-46.8 \mathrm{~km} \mathrm{~s}^{-1}$ and $-45.7 \mathrm{~km} \mathrm{~s}^{-1}$. In the images at $-44.6 \mathrm{~km} \mathrm{~s}^{-1},-43.6 \mathrm{~km} \mathrm{~s}^{-1}$, and $-42.5 \mathrm{~km} \mathrm{~s}^{-1}$ this emission extends to the western section of the bubble, where the IRDC SDS 341.194-0.221 is present. S 24 appears to be almost completely encircled by molecular gas in the images at $-43.6 \mathrm{~km} \mathrm{~s}^{-1}$ and $-42.5 \mathrm{~km} \mathrm{~s}^{-1}$, while at $-41.5 \mathrm{~km} \mathrm{~s}^{-1}$ and $-40.5 \mathrm{~km} \mathrm{~s}^{-1}$ the $\mathrm{CO}$ emission is present to the west of the bubble, coincident with the IRDC.

A close inspection of the emission in the interval from $-48.9 \mathrm{~km} \mathrm{~s}^{-1}$ to $-42.5 \mathrm{~km} \mathrm{~s}^{-1}$ reveals bright molecular emission linked to G341.217-0.237 and the eastern border of the IR bubble.

The CO emission associated with the different IR sources described above is analyzed in more detail in the following sections. Material linked to the IRDC SDC341.232-0.268 and present in the interval from $-49.5 \mathrm{~km} \mathrm{~s}^{-1}$ to $-42.1 \mathrm{~km} \mathrm{~s}^{-1}$ is being analyzed in detail by Vasquez et al. (in prep.).

\section{Joining the details}

In this section we investigate the gas distribution in S 24 in more detail and study the different features in its environment and its relation to the warm and cold dust and the YSO candidates.

\subsection{G341.220-0.213}

Panel A of Fig. 12 displays the ${ }^{13} \mathrm{CO}(3-2)$ emission with velocities in the range $-43.8 \mathrm{~km} \mathrm{~s}^{-1}$ and $-42.0 \mathrm{~km} \mathrm{~s}^{-1}$ superimposed onto the emission at $8 \mu \mathrm{m}$ for comparison. The emission distribution at these velocities reveals a molecular arc-like structure closely encircling the bright northern and western borders of G341.220-0.213. At $8 \mu \mathrm{m}, \mathrm{G} 341.220-0.213$, which resembles a horseshoe, is open opposite to the molecular emission. The IR source clearly interacts with the surrounding molecular gas and evolves in a medium with a density gradient. The coincidence of G341.220-0.213 with emission at $24 \mu \mathrm{m}$ (see Sect. 5) suggests excitation sources inside. Bearing in mind the morphology of the source at $8 \mu \mathrm{m}$ and in molecular lines, a champagne flow (Garay \& Lizano 1999) cannot be discarded.

A number of YSO candidates appear inside the horseshoe: one MSX source classified as MYSO (\#2 in Table 1); one Spitzer and one WISE source coincident in position, both classified as Class I (\#15 and \#34). We inspected the SED of sources \#2, \#15, and \#34, which are assumed to be only one source, using the online $^{4}$ tool developed by Robitaille et al. (2007), which can help to distinguish between evolved stars and reliable candidate YSOs. This tool fits radiation transfer models to observational data according to a $\chi^{2}$ minimization algorithm. Models that accomplished the following condition were selected:

$\chi^{2}-\chi_{\min }^{2}<3 n$,

where $\chi_{\min }^{2}$ is the minimum $\chi^{2}$ value from all models, and $n$ is the number of input data fluxes.

To perform the fitting, we used the photometric data listed in Table 1 along with fluxes derived from Herschel and ATLASGAL images, visual absorption values in the range 30-40 mag, and distances in the range from 3.2 to $4.2 \mathrm{kpc}$. The result is shown in the left panel of Fig. 13. Fluxes obtained from Herschel and ATLASGAL were considered upper limits. The best fit suggests a massive central source of about $10 M_{\odot}$, a disk mass of $5 \times 10^{-4} M_{\odot}$, an envelope mass of $21 M_{\odot}$, a total luminosity of $5300 L_{\odot}$, and an age of $1 \times 10^{6} \mathrm{yr}$. With these characteristics, the evolutionary stage of the central source according to Robitaille et al. (2007) would correspond to Stage II (objects with optically thick disks). These results point to a massive central object.

We propose that this source has started modifying its environs by dissociating and then ionizing the nearby material, generating a PDR detected by its emission at $8 \mu \mathrm{m}$ at the interface between the ionized and molecular material. It may have contributed to the heating of the dust as revealed by the emission at $24 \mu \mathrm{m}$. In addition, the detection of maser emission toward G341.220-0.213 (see Sect. 1) indicates that star formation is active.

A low-emission region is located at $\mathrm{RA}, \operatorname{Dec}(\mathrm{J} 2000)=$ $\left(16^{\mathrm{h}} 52^{\mathrm{m}} 22.5^{\mathrm{s}},-44^{\circ} 26^{\prime} 40^{\prime \prime}\right)$, probably linked to a weak IR arc seen slightly to the north of that position.

Panels $\mathrm{B}$ and $\mathrm{C}$ of Fig. 12 show the emission distribution in the ${ }^{13} \mathrm{CO}(2-1)$ and $\mathrm{C}^{18} \mathrm{O}(2-1)$ lines for the same velocity interval as panel $\mathrm{A}$, while in panel $\mathrm{D}$ the emission corresponds to the $\mathrm{CO}(2-1)$ line in a slightly different velocity interval. The emission distribution reveals a molecular clump coincident with the G341.220-0.213. No signs of the arc-like structure are present in the $(2-1)$ transitions, probably due to the lower angular resolution of these lines $\left(30^{\prime \prime}\right)$ in comparison with the one of the ${ }^{13} \mathrm{CO}(3-2)\left(20{ }^{\prime \prime}\right)$.

The molecular clump detected in the interval $[-44.0$, -42.0] $\mathrm{km} \mathrm{s}^{-1}$ coincides with the emission of cold dust as imaged in the far-IR by LABOCA (AGAL 341.217-00.212) and Herschel (see Fig. 4), indicating that gas and dust are well mixed in the region.

The analysis of the data cubes allows us to identify molecular gas associated with G341.220-0.213 in the velocity interval from $-46.5 \mathrm{~km} \mathrm{~s}^{-1}$ to $-40.2 \mathrm{~km} \mathrm{~s}^{-1}$.

4 http://caravan.astro.wisc.edu/protostars/ 


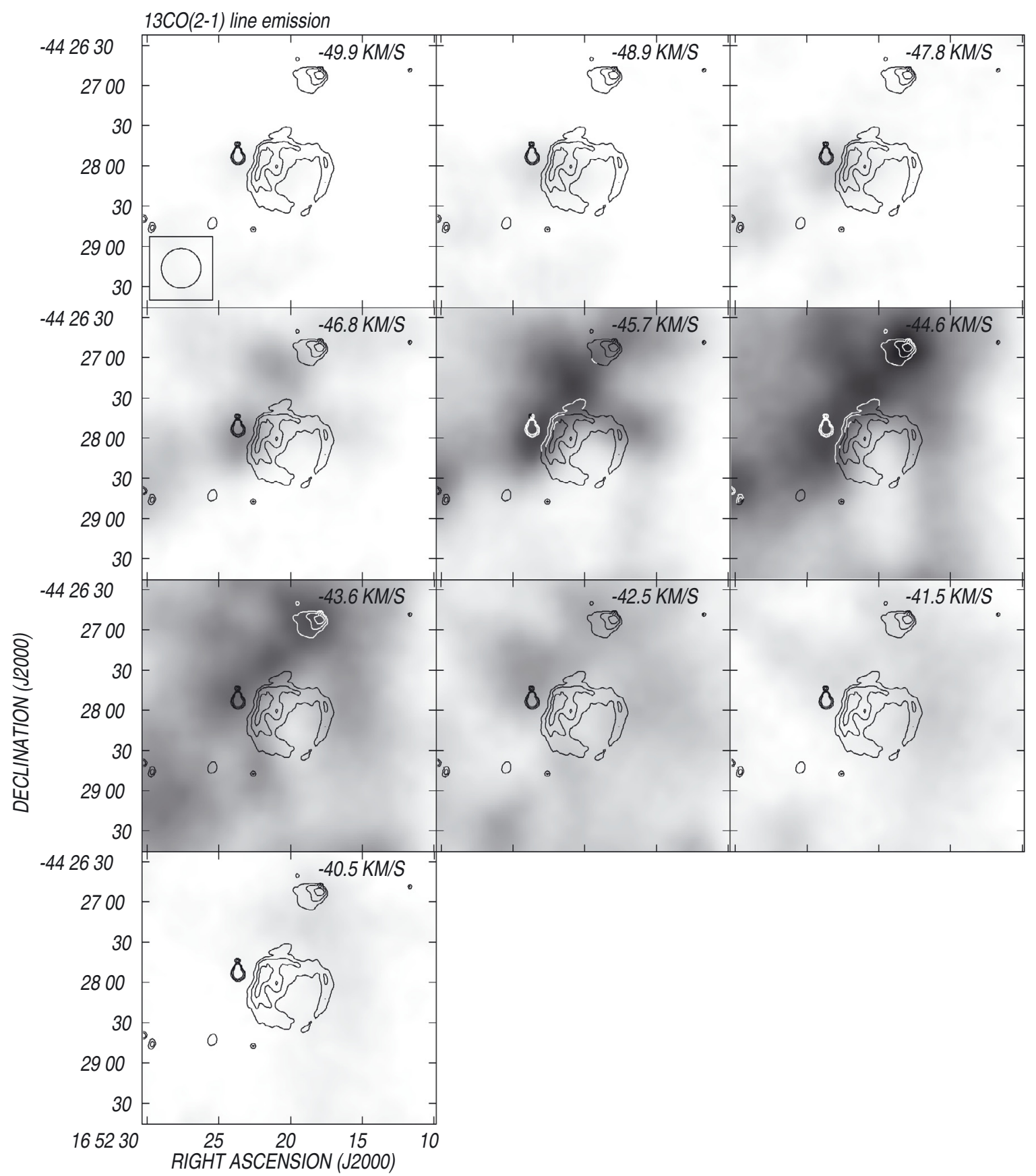

Fig. 11. Channel maps of the ${ }^{13} \mathrm{CO}(2-1)$ line (in grayscale) for the velocity interval from $-49.9 \mathrm{~km} \mathrm{~s}^{-1}$ to $-40.5 \mathrm{~km} \mathrm{~s}^{-1}$ in steps of $1.05 \mathrm{~km} \mathrm{~s}^{-1}$ overlaid onto the $8 \mu \mathrm{m}$ emission (in contours). The grayscale extends from $0.5 \mathrm{~K}$ to $17.0 \mathrm{~K}$.

\section{2. $H I I S 24$}

The upper left panel of Fig. 14 shows the ${ }^{13} \mathrm{CO}(3-2)$ emission distribution at $-43.2 \mathrm{~km} \mathrm{~s}^{-1}$ in grayscale and contours, while the upper right panel displays an overlay of the same CO contours and the emission at $8 \mu \mathrm{m}$. The middle and bottom panels show the emission at the same velocity for the ${ }^{13} \mathrm{CO}(2-1)$ and $\mathrm{C}^{18} \mathrm{O}(2-1)$ lines, respectively.

The emission at this velocity reveals a U-shaped, almost complete molecular shell encircling the S24 bubble. The strongest emission regions of this molecular shell encircle the brightest sections of $\mathrm{S} 24$ as seen at $8 \mu \mathrm{m}$. The shell appears open toward RA, Dec $(J 2000)=\left(16^{\mathrm{h}} 52^{\mathrm{m}} 19^{\mathrm{s}},-44^{\circ} 28^{\prime} 30^{\prime \prime}\right)$. The emission in the western part of the shell coincides with the IRDC SDC341.194-0.221, which borders this section of the bubble.
The molecular shell is extends throughout the interval from $-46 \mathrm{~km} \mathrm{~s}^{-1}$ to $-41.5 \mathrm{~km} \mathrm{~s}^{-1}$.

As shown in Fig. 11, in the velocity interval from $-46.5 \mathrm{~km} \mathrm{~s}^{-1}$ to $-43.5 \mathrm{~km} \mathrm{~s}^{-1}$ the molecular gas borders the eastern, northern, and western sections of S 24. Molecular gas at these velocities coincides with cold dust detected in the far IR at $\lambda>160 \mu \mathrm{m}$, as shown in Figs. 2 and 4. Molecular shells have been found around many IR dust bubbles, in particular those identified as HII regions (e.g., Petriella et al. 2010; Pomarès et al. 2009; Deharveng et al. 2010). The presence of CO in the HII region interior suggests that the shell corresponds to a three-dimensional structure. The appearance of the shell indicates that it is inhomogeneous, with the molecular gas distributed unisotropically (e.g., Anderson et al. 2015). 
A\&A 585, A30 (2016)

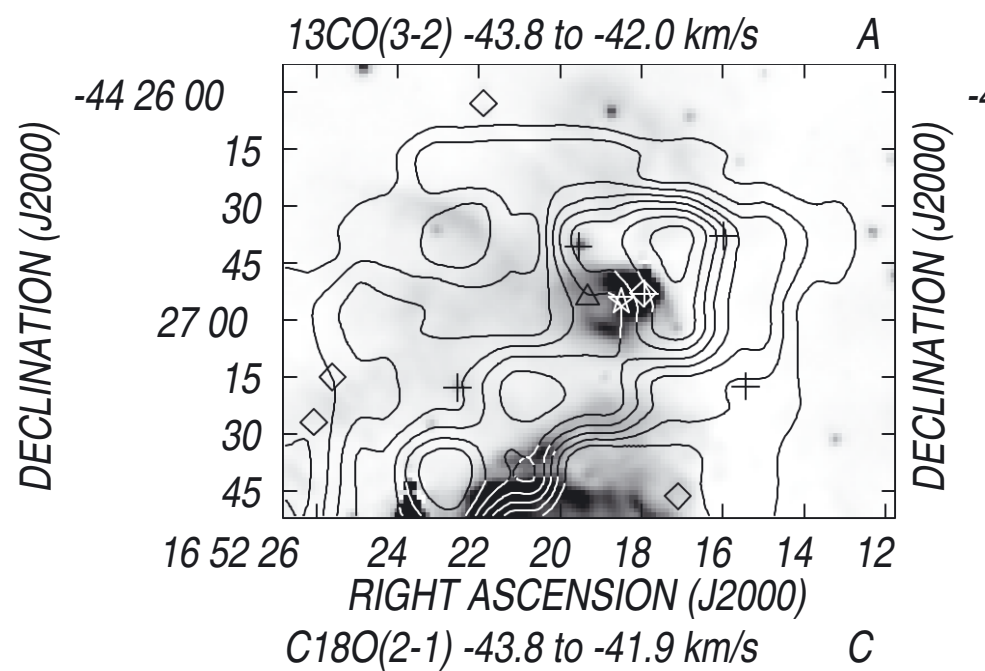

442600
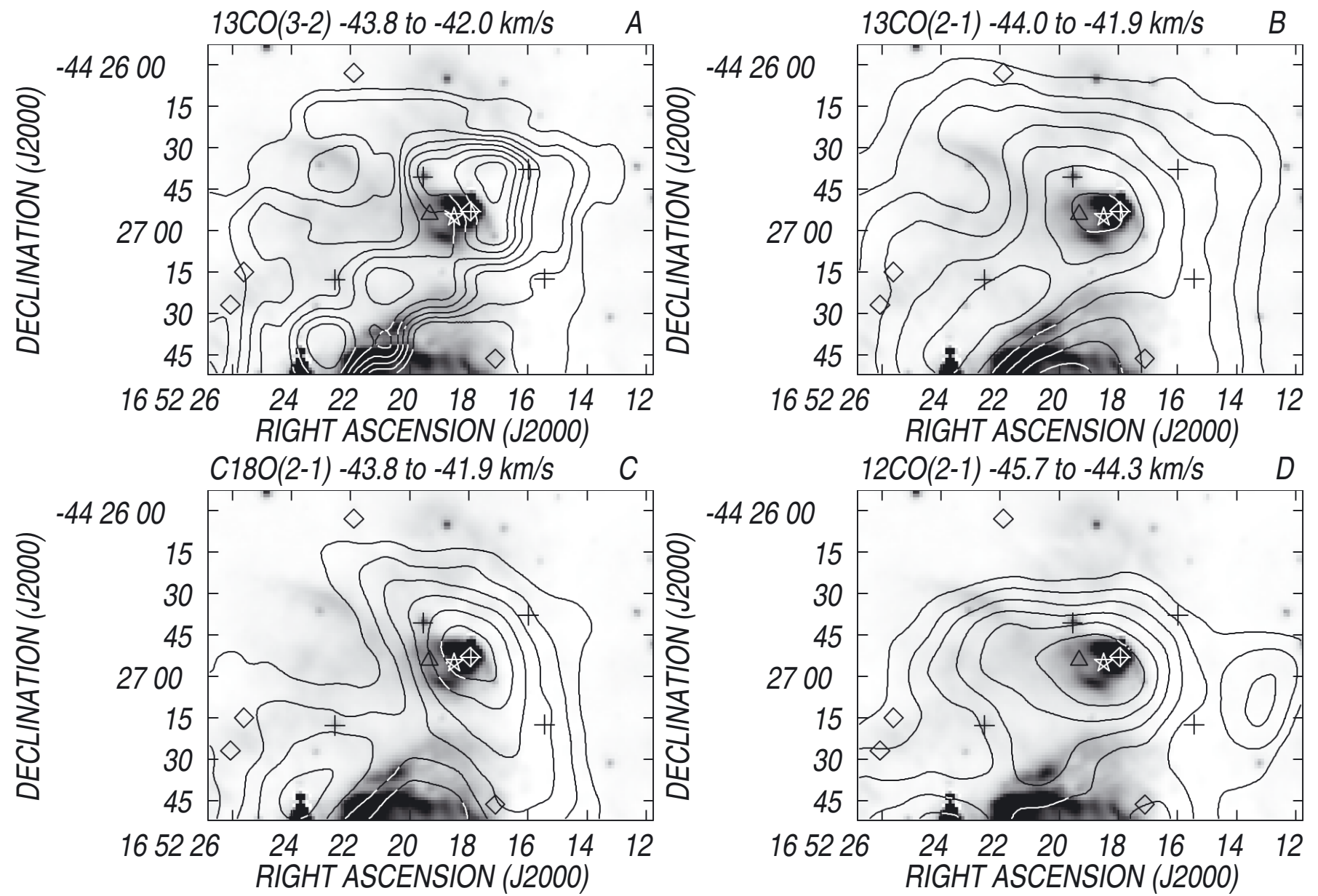

Fig. 12. Panels A)-D): overlay of the CO emission distribution in contours and the emission at $8 \mu \mathrm{m}$ in grayscale towards G341.220-0.213. Panel A): ${ }^{13} \mathrm{CO}(3-2)$ line emission in the interval from $-43.8 \mathrm{~km} \mathrm{~s}^{-1}$ to $-42.0 \mathrm{~km} \mathrm{~s}^{-1}$. Contours are from $5 \mathrm{~K}$ to $12 \mathrm{~K}\left(T_{\mathrm{mb}}\right)$ in steps of $1 \mathrm{~K}$. the different symbols mark the position of YSO candidates: stars: MSX sources, triangles: 2MASS sources, crosses: Spitzer sources, diamonds: WISE sources. Panel B): ${ }^{13} \mathrm{CO}(2-1)$ line emission in the interval from $-44.0 \mathrm{~km} \mathrm{~s}^{-1}$ to $-41.9 \mathrm{~km} \mathrm{~s}^{-1}$. Contours are from $5 \mathrm{~K}$ to $11 \mathrm{~K}\left(T_{\mathrm{mb}}\right)$ in steps of $1 \mathrm{~K}$. Panel C): $\mathrm{C}^{18} \mathrm{O}(2-1)$ line emission in the interval from $-43.8 \mathrm{~km} \mathrm{~s}^{-1}$ to $-41.9 \mathrm{~km} \mathrm{~s}^{-1}$. Contours are from $1.5 \mathrm{~K}$ to $4 \mathrm{~K}\left(T_{\mathrm{mb}}\right)$ in steps of $0.5 \mathrm{~K}$. Panel D): ${ }^{12} \mathrm{CO}(2-1)$ line emission in the interval from $-45.7 \mathrm{~km} \mathrm{~s}^{-1}$ to $-44.3 \mathrm{~km} \mathrm{~s}^{-1}$. Contours are from $9 \mathrm{~K}$ to $14 \mathrm{~K}\left(T_{\mathrm{mb}}\right)$ in steps of $1 \mathrm{~K}$.

The molecular shell might be expanding at $2-3 \mathrm{~km} \mathrm{~s}^{-1}$ due to the difference in pressure between the ionized gas inside the bubble and the neutral gas outside, although no signs of expansion are detected in position-velocity diagrams. We note, however, that low expansion velocities such as those expected in this case are difficult to detect. In the case of expansion, the emission detected toward the interior of the region would correspond to the approaching and receding sections of the shell.

Three YSO candidates are projected onto the $\mathrm{S} 24$ bubble: one MYSO (\# 1 in Table 1) and two WISE sources identified as Class II (\#35 and \#36).

A number of YSOs also coincide in position with the molecular shell, suggesting that star formation is active in the environs of the bubble.

Two YSOs appear projected onto the IRDC SDC341.1940.221 . They do not coincide with the spot of maser emission described in Sect. 1 projected onto this IRDC.

In addition to these YSO candidates, three stars could be identified in the 2MASS catalog in the region under study. These stars are indicated with green asterisks in the $\left[H-K_{\mathrm{s}}\right.$, $J-H]$ and $\left[H-K_{\mathrm{S}}, K_{\mathrm{S}}\right]$ diagrams of Fig. 3. In particular, 2MASS J17502070-4428012 is projected inside the bubble at RA, Dec $(J 2000)=\left(16^{\mathrm{h}} 52^{\mathrm{m}} 20.7^{\mathrm{s}},-44^{\circ} 28^{\prime} 1^{\prime \prime}\right.$ '. $)$. Its locus in the $\mathrm{CC}$ and CM diagrams $\left(H-K_{\mathrm{S}}\right)=1.19 \mathrm{mag},(J-H)=2.18 \mathrm{mag}$, and $K_{\mathrm{s}}=10.94 \mathrm{mag}$ ) is indicative of a massive star with an optical absorption $A_{\mathrm{V}} \simeq 30 \mathrm{mag}$. Although more studies are necessary, this star might be responsible for dissociating and ionizing the gas, creating the observed PDR at $8 \mu \mathrm{m}$.

\subsection{Interface between S24 and G341.217-0.237}

As described in Sect. 6, a bright molecular clump appears projected onto the interface between G341.217-0.237 and S 24 at RA, Dec $(J 2000)=\left(16^{\mathrm{h}} 52^{\mathrm{m}} 23^{\mathrm{s}},-44^{\circ} 28^{\prime}\right)$. Figure 15 displays an enlargement of the region of the clump showing the emission in the ${ }^{13} \mathrm{CO}(3-2)$ line at $-47.4 \mathrm{~km} \mathrm{~s}^{-1}$ (in contours) and the $8 \mu \mathrm{m}$ image in grayscale. The peak $\mathrm{CO}$ emission is coincident with the interface between the two sources. The anticorrelation between the $\mathrm{CO}$ and IR emissions strongly suggests that the molecular clump has modeled the eastern border of the PDR in the HII region.

These facts suggest that S24 and G341.217-0.237 are interacting with the molecular clump. The molecular clump coincides with emission at $870 \mu \mathrm{m}$ and in the Herschel bands at 250 and $350 \mu \mathrm{m}$.

The YSO candidate \# 43, classified as Class I, coincides with G341.217-0.237. The SED corresponding to this source is shown in the right panel of Fig. 13. To perform the fitting we used the 

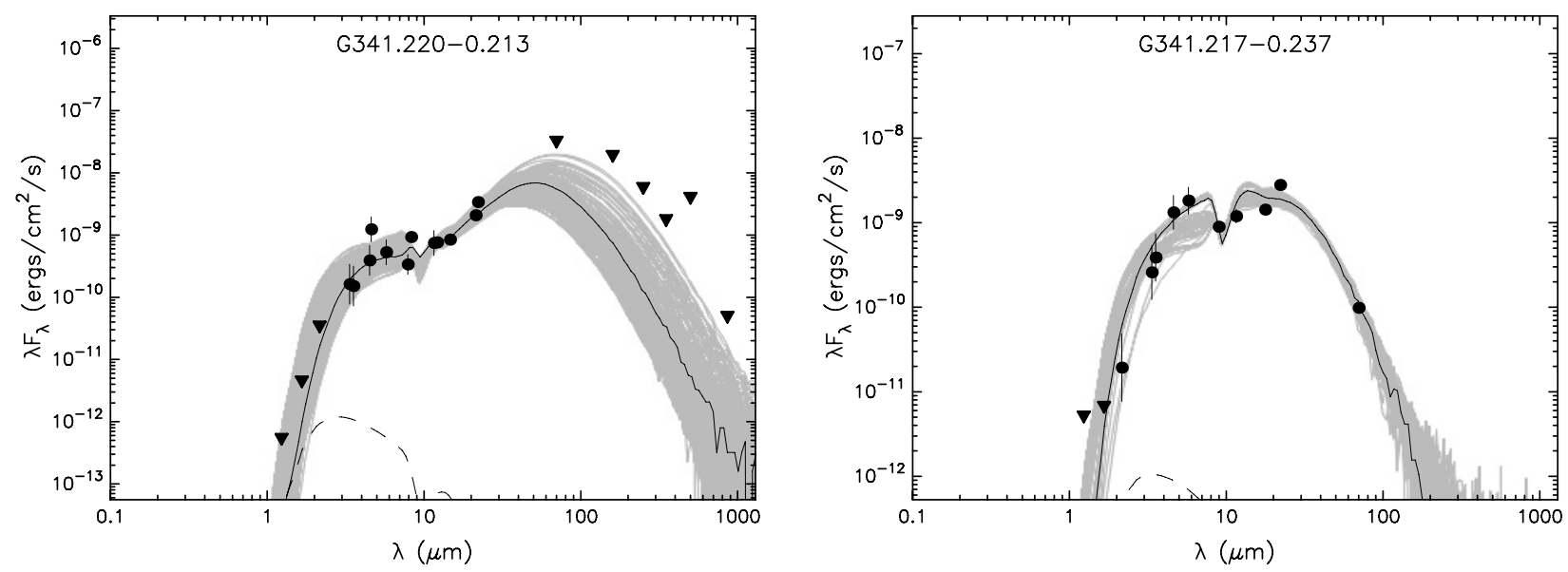

Fig. 13. Left panel: SED of YSOs from Table 1 in G341.220-0.213. Input data are indicated by filled circles. The triangles indicate upper limits. The black line corresponds to the best fit. The fittings that obey Eq. (1) appear indicated by gray lines. The dashed line shows the emission of the stellar photosphere including foreground interstellar extinction. Right panel: SED of source \#43 from Table 1 in G341.217-0.237.

photometric data listed in Table 1 and the flux derived from the image at $70 \mu \mathrm{m}$. Herschel fluxes at longer wavelengths include a significant part of the $\mathrm{S} 24$ bubble and were not used for the SED. Visual absorption is in the range 30-50 mag, and distances are from 3.2 to $4.2 \mathrm{kpc}$. The best fit corresponds to a central source of about $15 M_{\odot}$, a disk mass of $8 \times 10^{-3} M_{\odot}$, an envelope mass of $1.1 \times 10^{-5} M_{\odot}$, a total luminosity of $22000 L_{\odot}$, and an age of $1.3 \times 10^{6} \mathrm{yr}$. According to this fitting, the object is probably in Stage III (objects with optically thin disks). However, as pointed out by Deharveng et al. (2012), Offner et al. (2012), and Robitaille (2008), these results should be taken with caution. The presence of emission at $24 \mu \mathrm{m}$ coincident with G341.217-0.237 suggests that the ionizing photons from the massive source heat the dust and probably dissociate and ionize the molecular gas.

\section{Parameters of the molecular gas}

The molecular mass linked to G341.220-0.213, the clump at the interface between G341.217-0.237 and S 24 and the S 24 HII region, and the molecular mass in the whole region were estimated from the emission in the $J=2 \rightarrow 1$ line. The derived parameters are listed in Table 2.

Assuming local thermodinamic equilibrium (LTE) conditions and that the emission in the $\mathrm{CO}(2-1)$ line is optically thick, we derived the excitation temperature $T_{\text {exc }}$ from the emission in the $\mathrm{CO}(2-1)$ line using

$T_{\mathrm{p}-12 \mathrm{CO}}=T_{12 \mathrm{CO}}^{*}\left[\left(\mathrm{e}^{\frac{T_{12}^{*}}{T_{\mathrm{exc}}}}-1\right)^{-1}-\left(\mathrm{e}^{\frac{T_{12}^{*}}{T_{\mathrm{bg}}}}-1\right)^{-1}\right]$,

where $T_{12}^{*}=h v_{12} / k, v_{12}$ the frequency of the ${ }^{12} \mathrm{CO}(2-1)$ line, and $T_{\mathrm{bg}}=2.7 \mathrm{~K}$. To obtain the peak main beam temperature in the $\mathrm{CO}(2-1)$ line $\left(T_{\mathrm{p}-12 \mathrm{CO}}\right)$ we took into account the averaged spectrum within regions of about $30^{\prime \prime}$ in diameter for the cases of G341.220-0.213 and the interface between G341.217-0.237 and $\mathrm{S} 24$, while for the S 24 region and the whole region the spectra were averaged in a region of $2^{\prime}$ and $3^{\prime}$ in diameter, respectively.

The optical depth $\tau_{13}$ was obtained from the ${ }^{13} \mathrm{CO}(2-1)$ line by assuming that the excitation temperature is the same for $\mathrm{CO}(2-1)$ and ${ }^{13} \mathrm{CO}(2-1)$ emission lines using the expression

$\tau_{13}=-\ln \left[1-\frac{T_{\mathrm{p}-\mathrm{mb}}\left({ }^{13} \mathrm{CO}\right)}{T_{13}^{*}}\left[\left(\mathrm{e}^{\frac{T_{13}^{*}}{T_{\mathrm{exc}}}}-1\right)^{-1}-\left(\mathrm{e}^{\frac{T_{13}^{*}}{T_{\mathrm{bg}}}}-1\right)^{-1}\right]^{-1}\right]$, where $T_{13}^{*}=h v_{13} / k, v_{13}$ the frequency of the ${ }^{13} \mathrm{CO}(2-1)$ line. Assuming LTE, the ${ }^{13} \mathrm{CO}$ column density, $N_{\text {tot }}\left({ }^{13} \mathrm{CO}\right)$, can be estimated from the ${ }^{13} \mathrm{CO}(2-1)$ line data following

$N_{\text {tot }}\left({ }^{13} \mathrm{CO}\right)=3.2 \times 10^{14}\left[\frac{\mathrm{e}^{T_{13}^{*} / T_{\text {exc }}}}{1-\mathrm{e}^{-T_{13}^{*} / T_{\text {exc }}}}\right] T_{\text {exc }} \int \tau^{13} \mathrm{~d} v\left(\mathrm{~cm}^{-2}\right)$.

The integral of Eq. (6) can be approximated by

$T_{\operatorname{exc}} \int \tau^{13} \mathrm{~d} v \approx \frac{\tau^{13}}{1-\mathrm{e}^{\left(-\tau^{13}\right)}} \int T_{\mathrm{mb}} \mathrm{d} v$

This approximation helps to eliminate to some extended optical depth effects and is good within $15 \%$ for $\tau<2$ (Rohlfs \& Wilson 2004). Bearing in mind the $\tau_{13}$-values listed in Table 2 , it is appropriate for our region. Then, the molecular mass was calculated using

$M\left(\mathrm{H}_{2}\right)=\left(m_{\text {sun }}\right)^{-1} \mu m_{\mathrm{H}} A N\left(\mathrm{H}_{2}\right) d^{2} \quad\left(M_{\odot}\right)$,

where $m_{\text {sun }}$ is the solar mass $\left(\sim 2 \times 10^{33} \mathrm{~g}\right), \mu$ is the mean molecular weight, which is assumed to be equal to 2.76 after allowing for a relative helium abundance of $25 \%$ by mass (Yamaguchi et al. 1999), $m_{\mathrm{H}}$ is the hydrogen atom mass $\left(\sim 1.67 \times 10^{-24} \mathrm{~g}\right)$, $A$ is the solid angle of the CO emission, $d$ is the adopted distance expressed in $\mathrm{cm}$, and $N\left(\mathrm{H}_{2}\right)$ is the $\mathrm{H}_{2}$ column density, obtained using an abundance $N\left(\mathrm{H}_{2}\right) / N\left({ }^{13} \mathrm{CO}\right)=5 \times 10^{5}$ (Dickman 1978). Uncertainties in molecular masses and ambient densities are about $50 \%$ and $70 \%$, respectively, and originate mainly in distance uncertainties and optically thick emission in the ${ }^{13} \mathrm{CO}(2-1)$ line.

To explore the questions of temperature and density on G341.220-0.213 and G341.217-0.237 in depth, we performed a large velocity gradient (LVG) analysis (Scoville \& Solomon 1973; Goldreich \& Kwan 1974) for radiative transfer of molecular emission lines. We used the $l v g$ task implemented as part of the MIRIAD ${ }^{5}$ package of SMA. For a given column density (normalized by the line width), this program estimates the line radiation temperature of a molecular transition as a function of the kinetic temperature $\left(T_{\mathrm{k}}\right)$ and the $\mathrm{H}_{2}$ volume density $\left(n_{\mathrm{H} 2}\right)$. We generated $50 \times 50$ model grids for the ${ }^{13} \mathrm{CO}(3-2)$,

5 http://www.cfa.harvard.edu/sma/miriad/packages/ 

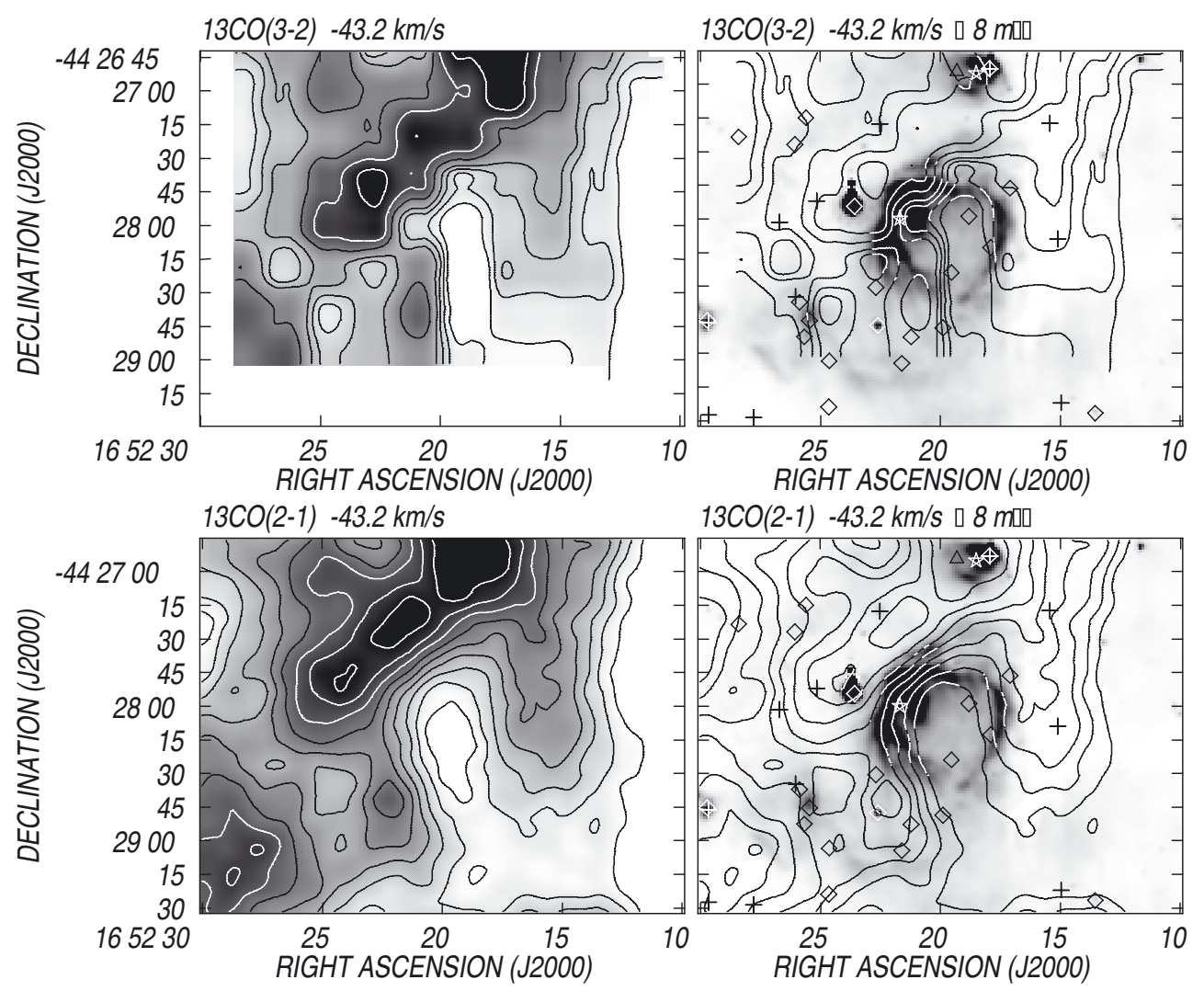

$13 \mathrm{CO}(2-1)-43.2 \mathrm{~km} / \mathrm{s}$ 口 $8 \mathrm{~m}$ [य

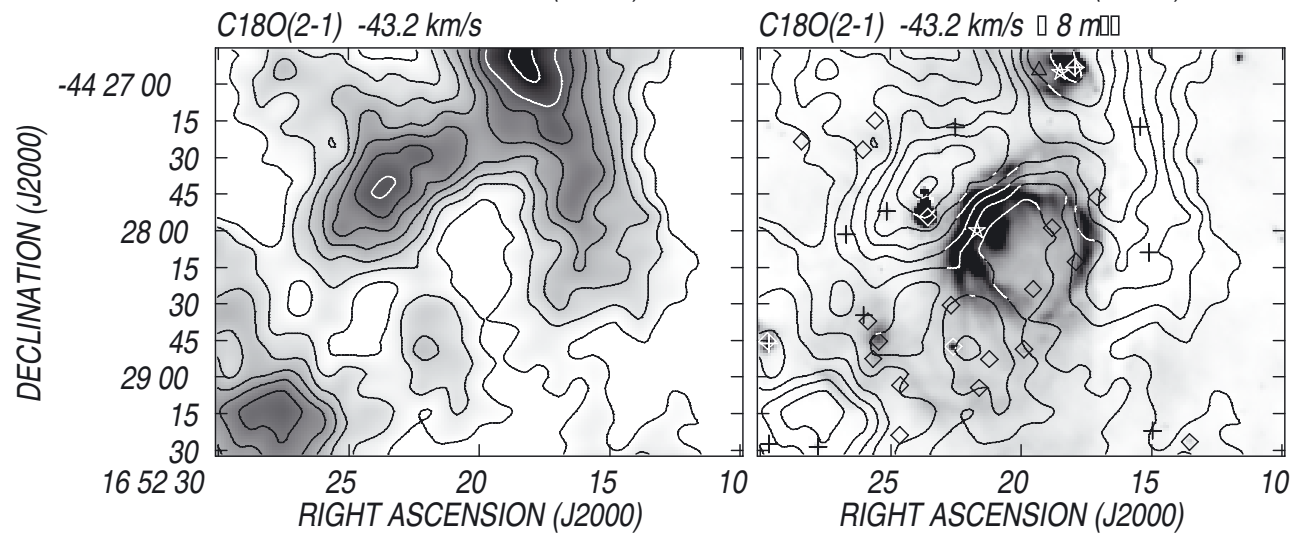

Fig. 14. Upper panels: left: ${ }^{13} \mathrm{CO}(3-2)$ emission distribution at $-43.2 \mathrm{~km} \mathrm{~s}^{-1}$ showing the molecular envelope around the $\mathrm{S} 24$ bubble. Contours are $3.0 \mathrm{~K}$ to $6.0 \mathrm{~K}\left(T_{\mathrm{mb}}\right)$ in steps of $1.0 \mathrm{~K}, 8.0 \mathrm{~K}, 10.0 \mathrm{~K}$, and $12.0 \mathrm{~K}$. Right: overlay of the same contours as in the left panel and the IRAC emission at $8 \mu \mathrm{m}$. The symbols have the same meaning as in Fig. 12. Middle panels: Left: ${ }^{13} \mathrm{CO}(2-1)$ emission distribution at $-43.2 \mathrm{~km} \mathrm{~s}^{-1}$. Contours are from $4.0 \mathrm{~K}$ to $12.0 \mathrm{~K}\left(T_{\mathrm{mb}}\right)$ in steps of $1.0 \mathrm{~K}$. Right: overlay of the same contours as in the left panel and the IRAC emission at $8 \mu \mathrm{m}$. Bottom panels: left: $\mathrm{C}^{13} \mathrm{O}(2-1)$ emission distribution at $-43.2 \mathrm{~km} \mathrm{~s}^{-1}$. Contours are from $1.0 \mathrm{~K}$ to $4.0 \mathrm{~K}\left(T_{\mathrm{mb}}\right)$ in steps of $0.5 \mathrm{~K}$, and $5 \mathrm{~K}$. Right: overlay of the same contours as in the left panel and the IRAC emission at $8 \mu \mathrm{m}$.

${ }^{13} \mathrm{CO}(2-1)$, and $\mathrm{CO}(2-1)$ lines with $T_{\mathrm{k}}$ in the range $1-300 \mathrm{~K}$ and $n_{\mathrm{H} 2}$ with the range $1 \times 10^{3}$ to $1 \times 10^{8} \mathrm{~cm}^{-3}$. In all cases we convolved the ${ }^{13} \mathrm{CO}(3-2)$ data with a Gaussian of FWHM $30^{\prime \prime}$ to smooth the cubes down to the angular resolution of the ${ }^{13} \mathrm{CO}(2-1)$ data. We then derived peak main beam temperatures and line widths for the studied components by fitting Gaussian functions to the line profiles. We then calculated two ratios, $R_{(3-2) /(2-1)}=T_{\mathrm{mb}}{ }^{13} \mathrm{CO}(3-2) / \mathrm{CO}(2-1)$ and $R_{12 / 13}=T_{\mathrm{mb}}{ }^{13} \mathrm{CO}(2-1) / \mathrm{CO}(2-1)$. The input column densities of $\mathrm{CO}$ and ${ }^{13} \mathrm{CO}$ were estimated from the dust-derived column density measured from the $870 \mu \mathrm{m}$ emission in each region, and assuming relative abundances $N(\mathrm{CO}) / N\left(\mathrm{H}_{2}\right)=1 \times 10^{-4}$ and $\mathrm{N}\left({ }^{13} \mathrm{CO}\right) / \mathrm{N}\left(\mathrm{H}_{2}\right)=2 \times 10^{-6}$ (Dickman 1978). The same procedure was applied to the IRDC SDC341.194-0.221, for which we did not derive molecular mass and ambient density since its molecular emission is included in the S 24 molecular shell.

Figure 16 shows the solutions for G341.220-0.213, G341.217-0.237, and the IRDC. They indicate that kinetic temperatures in the IRDC are about $20 \mathrm{~K}$, not different than derived in other IRDCs (Egan et al. 1998), and not much higher than typical temperatures of molecular gas without an extra heat source other than interstellar radiation field. The volume density of the IRDC is about $10^{4} \mathrm{~cm}^{-3}$. The temperatures for G341.220-0.213, G341.217-0.237 are slightly higher ( $30-40 \mathrm{~K})$, as expected, because they are active star-forming regions (see Sect. 7). Their volume densities are less well constrained, but values higher than $4 \times 10^{4} \mathrm{~cm}^{-3}$ are inferred from the plots. 


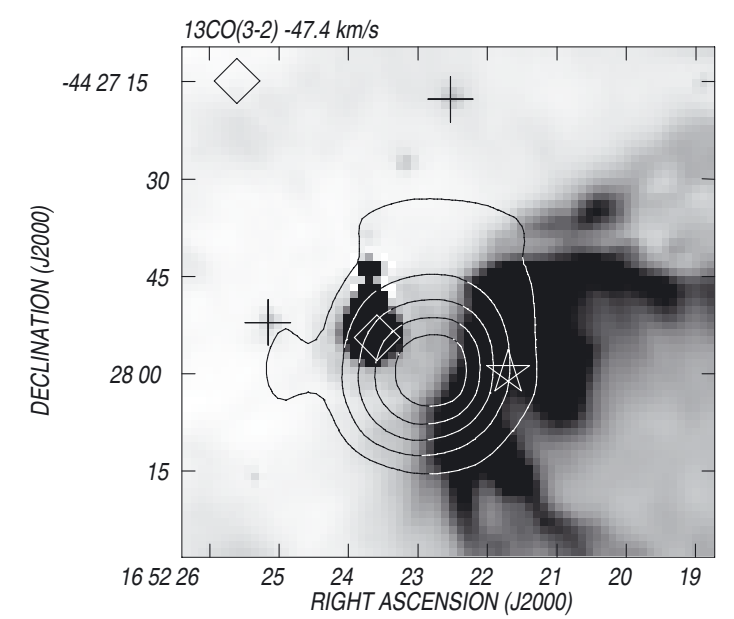

Fig. 15. Overlay of the ${ }^{13} \mathrm{CO}(3-2)$ emission distribution at $-47.4 \mathrm{~km} \mathrm{~s}^{-1}$ (in contours) and the emission at $8 \mu \mathrm{m}$ (in grayscale) showing the molecular clump at the interface between G341.217-0.237 and the $\mathrm{S} 24$ bubble. Contours are from $3 \mathrm{~K}$ to $6 \mathrm{~K}\left(T_{\mathrm{mb}}\right)$ in steps of $1 \mathrm{~K}$. The symbols are the same as in Fig. 12.

Table 2 shows that molecular gas densities are in the range $10^{4}-10^{5}$ for particular regions including the shell around the S 24 HII region. Masses derived from molecular data are compatible, within errors, with those obtained from the emission at $870 \mu \mathrm{m}$, assuming a gas-to-dust ratio equal to 100 . Additionally, molecular ambient densities are compatible with values derived from LVG analysis. The large difference for G341.217-0.237 arises because the mass estimate from molecular emission only corresponds to the emission at the interface between G341.2170.237 and the S 24 HII region. The ambient density obtained by distributing the total molecular mass in the whole observed region is high $\left(5900 \mathrm{~cm}^{-3}\right)$, indicating that G341.220-0.213, G341.217-0.237 and S 24 are evolving in a high-density ambient medium.

The high opacity estimated for G341.220-0.213 is expected considering the high column density of this source.

\section{Triggered star formation scenario in S 24?}

We have found evidence for ongoing star formation in the dense molecular envelope encircling the S $24 \mathrm{HII}$ region. Were these YSOs triggered by the collect-and-collapse (CC) mechanism? To answer this question, we can apply the analytical model by Whitworth et al. (1994). For the HII regions, the model predicts the age of the HII region at which the fragmentation occurs (the fragmentation timescale), $t_{\text {frag }}$, the size of the HII region at that moment, $R_{\text {frag }}$, the mass of the fragments, $M_{\text {frag }}$, and their separation along the compressed layer, $r_{\text {frag }}$. The parameters required to derive these quantities are the UV photon flux of the exciting star, $N_{\text {Ly }}$, the ambient density of the surrounding medium into which the HII region is evolving, $\mathrm{n}_{0}$, and the isothermal sound speed in the shocked gas, $a_{\mathrm{s}}$.

Since the exciting star is unknown, we took into account a wide range of spectral types, that is, from $\mathrm{O} 5 \mathrm{~V}$ to $\mathrm{O} 9 \mathrm{~V}$ stars, with UV fluxes in the range $N_{\mathrm{Ly}}=(18-0.8) \times 10^{48} \mathrm{~s}^{-1}$ (Martins et al. 2002). Using the mean $\mathrm{H}_{2}$ ambient density $n_{\mathrm{H} 2}=$ $5900 \mathrm{~cm}^{-3}$ (see Table 2), and $a_{\mathrm{s}}=0.2-0.6 \mathrm{~km} \mathrm{~s}^{-1}$, we obtained $t_{\text {frag }}=(6.6-8.8) \times 10^{5} \mathrm{yr}, R_{\text {frag }}=1.8-2.3 \mathrm{pc}, M_{\text {frag }}=22-29 M_{\odot}$, and, $r_{\text {frag }}=0.5-0.4 \mathrm{pc}$.
The dynamical age of the HII region can be estimated using the equation (Dyson \& Williams 1997)

$t_{\mathrm{dyn}}=\frac{4 R_{\mathrm{S}}}{7 c_{\mathrm{S}}}\left[\left(\frac{R}{R_{\mathrm{S}}}\right)^{7 / 4}-1\right]$,

where $R_{\mathrm{S}}$ is the original Strömgren radius, equal to $0.09-0.25 \mathrm{pc}$ for the adopted spectral types, and $c_{\mathrm{S}}$ is the sound velocity in the ionized gas. Derived dynamical ages span the range $(9-43) \times$ $10^{3} \mathrm{yr}$. We find that the dynamical age is younger than the fragmentation timescale $t_{\text {frag }}$ for the adopted ambient density. The comparison of these two quantities does not support a collectand-collapse process, which is a relatively slow process, for the triggering of star formation in the envelope. An RDI scenario could be investigated, but evidence of this process (such as pillars) appear to be absent. Very probably, the compact HII region is too young for triggering to have begun.

\section{Conclusions}

Based on the molecular emission in the $\mathrm{CO}(2-1),{ }^{13} \mathrm{CO}(2-1)$, $\mathrm{C}^{18} \mathrm{O}(2-1)$, and ${ }^{13} \mathrm{CO}(3-2)$ lines that were obtained with the APEX telescope and images in the near-, mid-, and far-IR from IRAC-Glimpse, Herschel, and ATLASGAL, we performed a multiwavelength analysis of the infrared dust bubble S 24 and two extended IR sources (G341.220-0.213 and G341.217-0.237) located in its environs. The region coincides with the IRAS point source 16487-4423, which is classified as UCHII. We also investigated the presence of YSO candidates in the region using the MSX, 2MASS, Spitzer, and WISE catalogs.

The molecular emission distribution shows that gas linked to the S 24 bubble, G341.220-0.213, and G341.217-0.237 has velocities between $-48.0 \mathrm{~km} \mathrm{~s}^{-1}$ and $-40.0 \mathrm{~km} \mathrm{~s}^{-1}$, compatible with the kinematical distance of $3.7 \mathrm{kpc}$ previously adopted for the region.

The gas distribution reveals a shell-like molecular structure of $\sim 0.8 \mathrm{pc}$ in radius detected in the velocity interval of $-46 \mathrm{~km} \mathrm{~s}^{-1}$ to $-41.5 \mathrm{~km} \mathrm{~s}^{-1}$, encircling the S 24 bubble. A cold dust counterpart of this shell is detected at wavelengths longer than $160 \mu \mathrm{m}$, that is, in the LABOCA and SPIRE images. In contrast, PACS emission at $70 \mu \mathrm{m}$ and MIPS emission at $24 \mu \mathrm{m}$ from warm dust appears to be projected inside the bubble. Dust temperatures derived from the emission at 70 and $160 \mu \mathrm{m}$ are in the range 26 to $60 \mathrm{~K}$, with the higher values closer to the center of the bubble. The detection of radio continuum emission and the warm dust indicate exciting sources and that the bubble is a compact $\mathrm{HII}$ region. We estimated a molecular gas and $\mathrm{H}_{2}$ ambient density in the shell of $3100 M_{\odot}$ and $2.5 \times 10^{4} \mathrm{~cm}^{-3}$. A search for excitation sources allowed identifying a massive star candidate projected onto the HII region, which would provide the ultraviolet photons to heat the gas and dissociate and ionize the gas. A number of YSO candidates appear to be projected onto the molecular shell.

Part of the molecular gas bordering the $\mathrm{S} 24$ bubble coincides with the extended IRDC SDC341.194-0.221, for which we derived a kinetic temperature of about $20 \mathrm{~K}$ and a volume density of $10^{4} \mathrm{~cm}^{-3}$, based on LVG analysis.

For the source G341.220-0.213, which resembles a horseshoe open toward the east, the ${ }^{13} \mathrm{CO}(3-2)$ line emission allowed detecting an arc-like structure with velocities in the range $-43.8 \mathrm{~km} \mathrm{~s}^{-1}$ to $-42.0 \mathrm{~km} \mathrm{~s}^{-1}$, encircling the brightest sections of the IR source. The morphology indicates that the molecular gas interacts with the IR source. A dust counterpart is detected in the Herschel (SPIRE and PACS) and LABOCA images. 

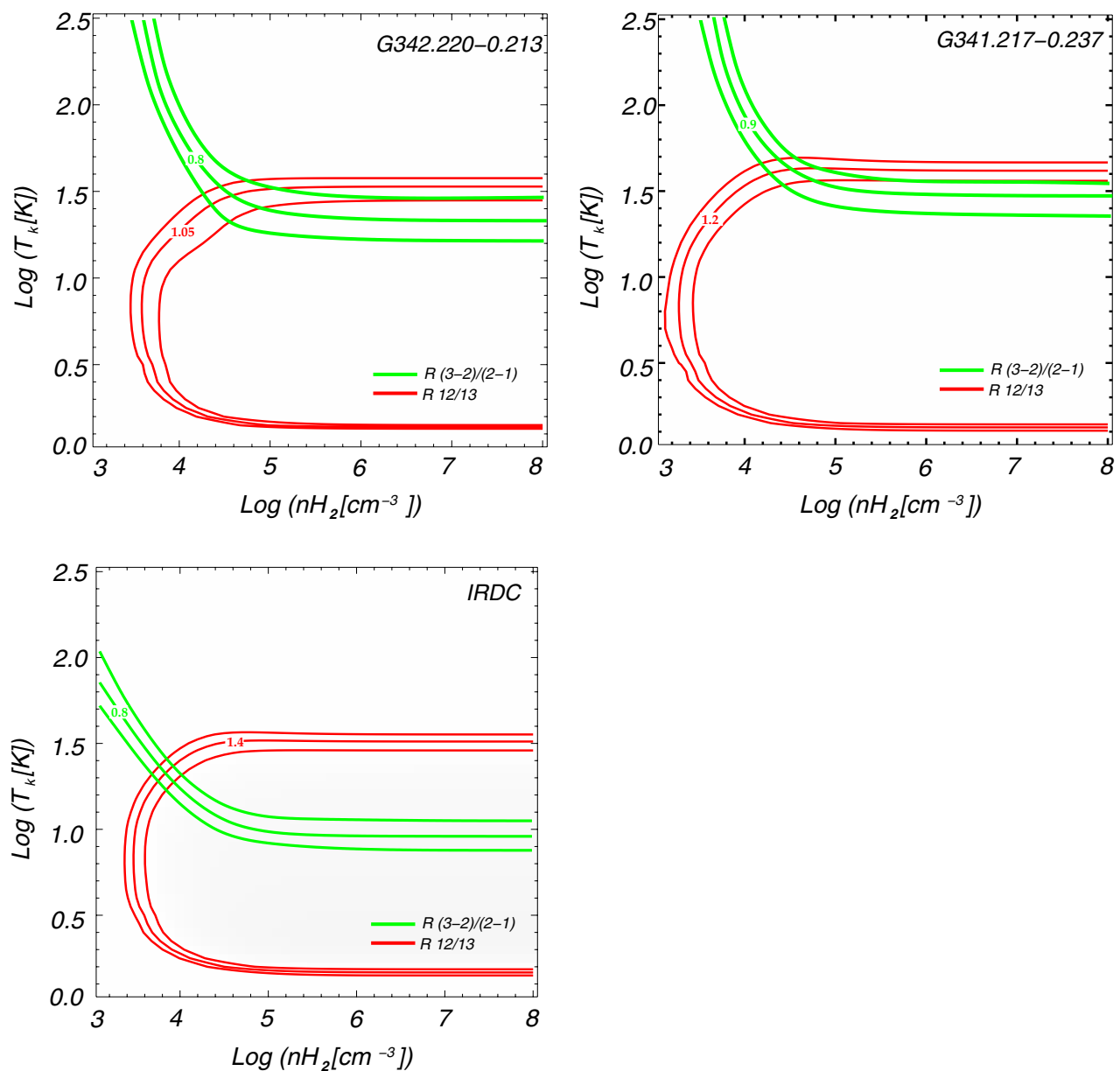

Fig. 16. LVG plots for G341.220-0.213, G341.217-0.237, and the IRDC SDC341.194-0.221. The green lines show the solutions for the ratios ${ }^{13} \mathrm{CO}(3-2) /{ }^{13} \mathrm{CO}(2-1)$, red lines those for ${ }^{13} \mathrm{CO}(2-1) / \mathrm{CO}(2-1)$. The central lines correspond to the observed ratios. The outer lines indicate $\sim 10 \%$ calibration uncertainties.

Table 2. Molecular gas parameters.

\begin{tabular}{lcccccccc}
\hline \hline & $\begin{array}{c}I\left({ }^{13} \mathrm{CO}\right) \\
{\left[\mathrm{K} \mathrm{km} \mathrm{s}^{-1}\right]}\end{array}$ & $\begin{array}{c}T_{\mathrm{exc}} \\
\tau_{13 \mathrm{CO}} \\
{[\mathrm{K}]}\end{array}$ & $\begin{array}{c}R \\
{\left[{ }^{\prime \prime}=\mathrm{pc}\right]}\end{array}$ & $\begin{array}{c}N\left({ }^{13} \mathrm{CO}\right) \\
{\left[10^{16} \mathrm{~cm}^{-2}\right]}\end{array}$ & $\begin{array}{c}N\left(\mathrm{H}_{2}\right) \\
{\left[10^{22} \mathrm{~cm}^{-2}\right]}\end{array}$ & $\begin{array}{c}M_{\text {mol }} \\
{\left[M_{\odot}\right]}\end{array}$ & $\begin{array}{c}n_{\mathrm{H}_{2}} \\
{\left[10^{4} \mathrm{~cm}^{-3}\right]}\end{array}$ \\
\hline G341.220-0.213 & 52.3 & 19.1 & 1.95 & $27=0.44$ & 14.6 & 7.3 & 1550 & 6.6 \\
Interface G341.217-0.237/S24 & 18.9 & 21.0 & 1.65 & $10=0.16$ & 5.2 & 2.6 & 470 & 41.6 \\
S 24-shell & 34.4 & 16.8 & 1.12 & $48=0.77$ & 7.3 & 3.6 & 3100 & 2.5 \\
Whole region & 32.4 & 16.1 & 1.14 & $114=1.8$ & 7.0 & 3.5 & 10300 & 0.59 \\
\hline
\end{tabular}

The spectral energy distribution constructed using Herschel and ATLASGAL fluxes allowed deriving a dust temperature of $28 \pm$ $1 \mathrm{~K}$. A molecular mass of $1550 M_{\odot}$ is linked to this source, compatible with the mass derived from ATLASGAL. The $\mathrm{H}_{2}$ ambient density amounts to $6.6 \times 10^{4} \mathrm{~cm}^{-3}$, in agreement with the ambient density value estimated from the LVG analysis. A number of IR point sources with characteristics of YSOs coincide with this region, which along with the maser emission indicates that star formation is active. The SED for the derived fluxes suggests a massive central source of $10 M_{\odot}$, which would be responsible for heating the dust and dissociating the molecular gas.

The source G341.217-0.237 also coincides with molecular gas and cold dust. The line emission allowed detecting a molecular clump at the interface between the S 24 HII region and G341.217-0.237, shaping the eastern border of the IR bubble. A high ambient density $\left(4.2 \times 10^{5} \mathrm{~cm}^{-3}\right)$ was estimated for the clump.
The total mass of molecular gas in the region and the $\mathrm{H}_{2}$ ambient density amount to $10300 M_{\odot}$ and $5900 \mathrm{~cm}^{-3}$, indicating that G341.220-0.213, G341.217-0.237, and the S 24 HII region are evolving into a high-density region.

Finally, we investigated whether a collect-and-collapse scenario can explain the YSOs in the dense envelope around the S 24 HII region. We conclude that neither this mechanism nor the RDI process seem to occur in this region. Very probably, the HII region is too young for triggering to have begun.

Acknowledgements. We acknowledge the anonymous referee for their comments. The ATLASGAL project is a collaboration between the Max-PlanckGesellschaft, the European Southern Observatory (ESO) and the Universidad de Chile. C.E.C. acknowledges the kind hospitality of M. Rubio and her family during her stay in Chile, and C. Kobulnicky for having suggested studying this object. L.C. acknowledges support from DIULS. V.F. acknowledges support from ESO-Chile Joint Committee and DIULS and would like to thank Ivan Valtchanov, Bruno Altieri, and Luca Conversi for their support and valuable assistance in Herschel data processing. V.F. also acknowledges support from the Faculty of the European Space Astronomy Centre (ESAC). This project was 
partially financed by CONICET of Argentina under project PIP 0356, UNLP under project 11/G120, and CONICyT of Chile through FONDECYT grant No. 1140839. This work is based [in part] on observations made with the Spitzer Space Telescope, which was operated by the Jet Propulsion Laboratory, California Institute of Technology under a contract with NASA. This publication makes use of data products from the Two Micron All Sky Survey, which is a joint project of the University of Massachusetts and the Infrared Processing and Analysis Center/California Institute of Technology, funded by the National Aeronautics and Space Administration and the National Science Foundation. The MSX mission was sponsored by the Ballistic Missile Defense Organization (BMDO).

\section{References}

Alexander, M. J., Kobulnicky, H. A., Kerton, C. R., \& Arvidsson, K. 2013, ApJ, 770,1

Allen, L. E., Calvet, N., D'Alessio, P., et al. 2004, ApJS, 154, 363

Anderson, L. D., Zavagno, A., Deharveng, L., et al. 2012, A\&A, 542, A10

Anderson, L. D., Deharveng, L., Zavagno, A., et al. 2015, ApJ, 800, 101

Bayandina, O. S., Val'Tts, I. E., \& Larionov, G. M. 2012, VizieR Online Data Catalog: J/AZh/89/611

Benjamin, R. A., Churchwell, E., Babler, B. L., et al. 2003, PASP, 115, 953

Bernard, J.-P., Paradis, D., Marshall, D. J., et al. 2010, A\&A, 518, L88

Brand, J., \& Blitz, L. 1993, A\&A, 275, 67

Bronfman, L., Nyman, L.-A., \& May, J. 1996, A\&AS, 115, 81

Cappa, C. E., Firpo, G. A., R., Rubio, M., \& Vasquez, J. 2013, Boletin de la Asociacion Argentina de Astronomia La Plata Argentina, 56, 311

Carey, S. J., Noriega-Crespo, A., Price, S. D., et al. 2005, in Amer. Astron. Soc. Meeting Abstr., BAAS, 37, 1252

Caswell, J. L., Fuller, G. A., Green, J. A., et al. 2010, MNRAS, 404, 1029

Churchwell, E., Povich, M. S., Allen, D., et al. 2006, ApJ, 649, 759

Churchwell, E., Watson, D. F., Povich, M. S., et al. 2007, ApJ, 670, 428

Contreras, Y., Schuller, F., Urquhart, J. S., et al. 2013, A\&A, 549, A45

Cutri, R. M., Skrutskie, M. F., van Dyk, S., et al. 2003, 2MASS All Sky Catalog of point sources

Cyganowski, C. J., Whitney, B. A., Holden, E., et al. 2008, AJ, 136, 2391

Deharveng, L., Zavagno, A., Schuller, F., et al. 2009, A\&A, 496, 177

Deharveng, L., Schuller, F., Anderson, L. D., et al. 2010, A\&A, 523, A6

Deharveng, L., Zavagno, A., Anderson, L. D., et al. 2012, A\&A, 546, A74

Dewangan, L. K., \& Ojha, D. K. 2013, MNRAS, 429, 1386

Dickman, R. L. 1978, ApJS, 37, 407

Dyson, J. E., \& Williams, D. A. 1997, The physics of the interstellar medium (Bristol: Institute of Physics Publishing)

Egan, M. P., Shipman, R. F., Price, S. D., et al. 1998, ApJ, 494, L199

Fazio, G. G., Hora, J. L., Allen, L. E., et al. 2004, ApJS, 154, 10

Garay, G., \& Lizano, S. 1999, PASP, 111, 1049

Goldreich, P., \& Kwan, J. 1974, ApJ, 189, 441

Griffin, M. J., Abergel, A., Abreu, A., et al. 2010, A\&A, 518, L3

Haverkorn, M., Gaensler, B. M., McClure-Griffiths, N. M., Dickey, J. M., \& Green, A. J. 2006, ApJS, 167, 230

Henning, T., Feldt, M., Stecklum, B., \& Klein, R. 2001, A\&A, 370, 100
Hildebrand, R. H. 1983, Quart. J. Roy. Astron. Soc., 24, 267

Koenig, X. P., Leisawitz, D. T., Benford, D. J., et al. 2012, ApJ, 744, 130

Larionov, G. M., \& Val'tts, I. E. 2008, VizieR Online Data Catalog: $\mathrm{J} / \mathrm{AZh} / 84 / 839$

Lumsden, S. L., Hoare, M. G., Oudmaijer, R. D., \& Richards, D. 2002, MNRAS, 336, 621

Martins, F., Schaerer, D., \& Hillier, D. J. 2002, A\&A, 382, 999

Mezger, P. G., \& Henderson, A. P. 1967, ApJ, 147, 471

Molinari, S., Swinyard, B., Bally, J., et al. 2010, A\&A, 518, L100

Murphy, T., Mauch, T., Green, A., et al. 2007, MNRAS, 382, 382

Offner, S. S. R., Robitaille, T. P., Hansen, C. E., McKee, C. F., \& Klein, R. I. 2012, ApJ, 753, 98

Ohlendorf, H., Preibisch, T., Gaczkowski, B., et al. 2013, A\&A, 552, A14

Ossenkopf, V., \& Henning, T. 1994, A\&A, 291, 943

Ott, S. 2010, in Astronomical Data Analysis Software and Systems XIX, eds. Y. Mizumoto, K.-I. Morita, \& M. Ohishi, ASP Conf. Ser., 434, 139

Peretto, N., \& Fuller, G. A. 2009, A\&A, 505, 405

Peretto, N., Fuller, G. A., Plume, R., et al. 2010, A\&A, 518, L98

Pestalozzi, M. R., Minier, V., \& Booth, R. S. 2005, A\&A, 432, 737

Petriella, A., Paron, S., \& Giacani, E. 2010, A\&A, 513, A44

Piat, M., Lagache, G., Bernard, J. P., Giard, M., \& Puget, J. L. 2002, A\&A, 393, 359

Poglitsch, A., Waelkens, C., Geis, N., et al. 2010, A\&A, 518, L2

Pomarès, M., Zavagno, A., Deharveng, L., et al. 2009, A\&A, 494, 987

Preibisch, T., Roccatagliata, V., Gaczkowski, B., \& Ratzka, T. 2012, A\&A, 541, A 132

Price, S. D., Egan, M. P., Carey, S. J., Mizuno, D. R., \& Kuchar, T. A. 2001, AJ, 121, 2819

Robitaille, T. P. 2008, in Massive Star Formation: Observations Confront Theory, eds. H. Beuther, H. Linz, \& T. Henning, ASP Conf. Ser., 387, 290

Robitaille, T. P., Whitney, B. A., Indebetouw, R., \& Wood, K. 2007, ApJS, 169, 328

Rohlfs, K., \& Wilson, T. L. 2004, Tools of Radioastronomy (Berlin-Heidelberg: Springer-Verlag)

Russeil, D., \& Castets, A. 2004, A\&A, 417, 107

Samal, M. R., Zavagno, A., Deharveng, L., et al. 2014, A\&A, 566, A122

Schuller, F., Menten, K. M., Contreras, Y., et al. 2009, A\&A, 504, 415

Scoville, N. Z., \& Solomon, P. M. 1973, ApJ, 180, 55

Siringo, G., Kreysa, E., Kovács, A., et al. 2009, A\&A, 497, 945

Urquhart, J. S., Moore, T. J. T., Schuller, F., et al. 2013, MNRAS, 431, 1752

Vassilev, V., Meledin, D., Lapkin, I., et al. 2008, A\&A, 490, 1157

Voronkov, M. A., Caswell, J. L., Ellingsen, S. P., Green, J. A., \& Breen, S. L. 2014, MNRAS, 439, 2584

Walsh, A. J., Purcell, C. R., Longmore, S. N., et al. 2014, MNRAS, 442, 2240

Watson, C., Povich, M. S., Churchwell, E. B., et al. 2008, ApJ, 681, 1341

Watson, C., Hanspal, U., \& Mengistu, A. 2010, ApJ, 716, 1478

Whitworth, A. P., Bhattal, A. S., Chapman, S. J., Disney, M. J., \& Turner, J. A. 1994, MNRAS, 268, 291

Wood, D. O. S., \& Churchwell, E. 1989, ApJ, 340, 265

Wright, E. L., Eisenhardt, P. R. M., Mainzer, A. K., et al. 2010, AJ, 140, 1868

Yamaguchi, R., Akira, M., \& Yasuo, F. 1999, in Star Formation 1999, ed. T. Nakamoto, 383 